\title{
Spectral Conditions for Stability and Stabilization of Positive Equilibria for a Class of Nonlinear Cooperative Systems
}

Precious Ugo Abara, Francesco Ticozzi and Claudio Altafini

The self-archived postprint version of this journal article is available at Linköping University Institutional Repository (DiVA):

http:/ / urn.kb.se/ resolve?urn=urn:nbn:se:liu:diva-145240

N.B.: When citing this work, cite the original publication.

Abara, P. U., Ticozii, F., Altafini, C., (2018), Spectral Conditions for Stability and Stabilization of Positive Equilibria for a Class of Nonlinear Cooperative Systems, IEEE Transactions on Automatic Control, 63(2), 402-417. https:// doi.org/ 10.1109/TAC.2017.2713241

Original publication available at:

https:/ / doi.org/ 10.1109/TAC.2017.2713241

Copyright: Institute of Electrical and Electronics Engineers (IEEE)

http:// www.ieee.org/ index.html

(C) 2018 IEEE. Personal use of this material is permitted. However, permission to reprint/ republish this material for advertising or promotional purposes or for creating new collective works for resale or redistribution to servers or lists, or to reuse any copyrighted component of this work in other works must be obtained from the IEEE. 


\title{
Spectral conditions for stability and stabilization of positive equilibria for a class of nonlinear cooperative systems
}

\author{
Precious Ugo Abara, Francesco Ticozzi and Claudio Altafini
}

\begin{abstract}
Nonlinear cooperative systems associated to vector fields that are concave or subhomogeneous describe well interconnected dynamics that are of key interest for communication, biological, economical and neural network applications. For this class of positive systems, we provide conditions that guarantee existence, uniqueness and stability of strictly positive equilibria. These conditions can be formulated directly in terms of the spectral radius of the Jacobian of the system. If control inputs are available, then it is shown how to use state feedback to stabilize an equilibrium point in the interior of the positive orthant.
\end{abstract}

Index terms - Nonlinear Cooperative Systems; Positive equilibrium points; Concave Systems; Subhomogeneous systems; Stability and Stabilization.

\section{INTRODUCTION}

Positive nonlinear systems are widely used as models of dynamical systems in which the state variables represent intrinsically non-negative quantities, such as concentrations, masses, populations, probabilities, etc. [13], [22], [31]. They are used for instance to model biochemical reactions [33], gene regulatory networks [24], population dynamics [15], epidemic processes [26], [21], compartmental systems [18], economic systems [22], hydrological networks, power control in wireless networks [11], [36], [10], certain types of neural networks [17], [14] and many other systems.

The aim of this paper is to investigate the existence, uniqueness, stability and stabilizability properties of positive equilibria of certain classes of nonlinear positive systems recurrent in applications. The main assumptions we make on our systems is that they are i) cooperative and ii) concave (or subhomogeneous). For example, in wireless networks, most power control algorithms assume that the (nonlinear) "interference functions" are scalable [36] (i.e., subhomogeneous [5], [9]). In a completely different field, the (nonlinear) "activation function" of a Hopfield-type neural network [17], [14] is often monotone and sigmoidal [14], [37], which means that it lacks inflection points once it is restricted to positive values. In gene regulatory network theory, the cooperative case appears as a special case (all activatory links), and asymptotic stability is achieved making use of saturated monotonicities such as Michaelis-Menten functional forms [24].

Work supported in part by a grant from the Swedish Research Council (grant n. 2015-04390 to C.A.). Preliminary versions of this manuscript were presented at the 54th and 55th Conf. on Decision and Control, see [34], [35]

P. Ugo Abara was with the Dept. of Information Engineering, via Gradenigo $6 \mathrm{~B}$, University of Padova, 35131, Padova, Italy. He is now with the Technical University of Munich, Germany.

F. Ticozzi is with the Dept. of Information Engineering, via Gradenigo 6B, University of Padova, 35131, Padova, Italy and the Physics and Astronomy Dept., Dartmouth College, 6127 Wilder, Hanover, NH (USA).

C.Altafini is with the Division of Automatic Control, Dept. of Electrical Engineering, Linköping University, SE-58183, Linköping, Sweden. email: claudio.altafini@liu.se
While the assumption of cooperativity is standard in the context of nonlinear positive systems [31], [1], [7], [27], as it guarantees invariance in the positive orthant, concavity corresponds to vector fields that "decline" when the state grows, and in such a way it favours the boundedness of the trajectories. Concavity appears naturally as a common feature in all examples mentioned above: subhomogeneity of order 1 is a proxy for concavity, sigmoidal functions are concave when restricted to the positive semiaxis, and so are MichaelisMenten functions. Combining the monotonic behavior of cooperative systems (with its lack of limit cycles and the relation of order it implies on the trajectories [31]) with the boundedness induced by concavity helps in achieving uniqueness and asymptotic stability of the equilibrium point. In fact, the asymptotic behavior of cooperative concave systems has been known for more than thirty years [32]. In particular, it is known that they admit a "limit set trichotomy" [23], i.e., three possible types of asymptotic behaviors: i) convergence to the origin; ii) convergence to a unique positive fixed point; iii) divergence to $\infty$, see [32], [30], [23], [31]. In [32], conditions for distinguishing between the first two cases are given, based on the spectral radius of the Jacobian. Alternatively, nonlinear versions of the Perron-Frobenius theorem for positive cones can be used to show "ray convergence" in a special metric, variously called part metric or Hilbert projective metric [6], [23]. The limit set trichotomy can all be placed along this ray.

In this paper we follow the approach of [32] and look at spectral conditions on the Jacobian of the system in order to describe the convergence to the two stable situations of the limit set trichotomy. In particular, we obtain novel sufficient conditions that guarantee the existence of strictly positive equilibria for monotone concave systems, but also for the more general class of monotone sub-homogeneous systems [5] and for that of monotone contractive systems [10].

It is worth remarking that in all the applications mentioned above the equilibrium point is normally required to be positive. In fact, the origin is typically not very interesting as an equilibrium: for example, a power control algorithm that converges to zero power is meaningless, and similarly for the other applications. A common trick to move the equilibrium point from the origin to the interior of the positive orthant is to add a non-vanishing positive input, usually a constant, to the system dynamics (a current in a neural network, a noise power in a wireless interference function, a constant mRNA synthesis rate in a gene network) [7], [27]. This trick is standard (and necessary) in linear positive systems [8], but not strictly necessary in the nonlinear case. As a matter of fact, in some cases the extra constant term seems more artificially motivated by the need of guaranteeing positivity of the equilibrium, rather than emerging from the problem setting. The conditions 
we provide in this paper do not require the use of additive external inputs to shift the stable equilibrium from the origin.

For concave systems, the properties of uniqueness and global attractivity of a positive equilibrium correspond to a bound on the spectral radius of the Jacobian at the origin, plus an additional condition that has to hold inside the positive orthant. When the concave nonlinearities are also bounded, the spectral radius of the Jacobian at the origin alone decides all the global dynamical features of the system [32].

Our conditions extend those provided in [32] is several directions. For instance, we show in the paper that when feedback design makes sense, then simple linear diagonal feedback can be used to choose any type of behavior in the trichotomy of possible asymptotic characters.

As another improvement with respect to [32], we show that our results hold essentially unchanged when we replace concave functionals with the broader class of subhomogeneous functionals. This is another class for which stability has been studied mostly at the origin [5], [9], or in presence of constant additive terms [11], [36]. The existence of simple spectral characterizations for uniqueness and stability of equilibria is not limited to concave/subhomogeneous vector fields: the c-contractive vector fields of [10] (which lead to a similar single global attractor) also admit an easy characterization in terms of the spectral radius of the Jacobian. The infinitesimal contractivity condition we obtain, i.e. that the spectral radius is always less than 1 , extends to the nonlinear case an equivalent property of linear interference functions [28], and leads to a Jacobian characterization similar to the one commonly used in contraction analysis of nonlinear systems [25]. Notice that the approach of [25] (which is based on contractions in Riemannian norm, not in Hilbert projective norm) cannot be used for concave/subhomogeneous systems passing through the origin, like those studied in this paper.

Lastly, it is worth remarking that cooperative dynamics can be often described by interconnected systems [19], [29], as it is indeed the case for the examples mentioned above. While the conditions we give in this paper are valid for general cooperative systems, when they are applied to the subclass of interconnected systems they can be sharpened and simplified, for instance expressed in terms of the spectral radius of the (constant) connectivity matrix alone. Furthermore, we show in the paper that for interconnected systems the stabilizing feedback design can be rendered distributed (i.e., requiring only information on the first neighbours of a node).

The rest of this paper is organized as follows: Section II introduces the necessary prerequisites of linear algebra and positive dynamical systems theory, including the existence and uniqueness criteria that we will need. For concave systems, the spectral characterizations of uniqueness and stability of the equilibria are given in Section III. They are generalized to subhomogeneous and contractive vector fields in Section IV. Stabilizability conditions via diagonal state feedback are given in Section V, while in Section VI the results are specialized to interconnected systems and applied to relevant examples. All proofs are gathered in the Appendix.

While parts of the results (in particular those of Sect. III and IV) have appeared in the conference papers [34], [35],
Sect. V and most of Sect. VI are novel material.

\section{Preliminary MATERial}

\section{A. Notation and linear algebra}

Throughout this paper $\mathbb{R}_{+}^{n}$ denotes the positive orthant of $\mathbb{R}^{n}$, int $\left(\mathbb{R}_{+}^{n}\right)$ its interior, and bd $\left(\mathbb{R}_{+}^{n}\right)=\mathbb{R}_{+}^{n} \backslash \operatorname{int}\left(\mathbb{R}_{+}^{n}\right)$ its boundary. If $x_{1}, x_{2} \in \mathbb{R}^{n}, x_{1} \leqslant x_{2}$ means $x_{1, i} \leqslant x_{2, i} \forall i=$ $1, \ldots, n\left(x_{1, i}=i\right.$-th component of $\left.x_{1}\right)$, while $x_{1}<x_{2}$ means $x_{1, i}<x_{2, i} \forall i=1, \ldots, n$, A matrix $A=\left[a_{i j}\right] \in \mathbb{R}^{n \times n}$ is said nonnegative (in the following indicated $A \geqslant 0$ ) if $a_{i j} \geqslant 0$ $\forall i, j$, and Metzler if $a_{i j} \geqslant 0 \forall i \neq j . A$ is irreducible if $\nexists \mathrm{a}$ permutation matrix $\Pi$ that renders it block diagonal:

$$
\Pi^{T} A \Pi=\left[\begin{array}{cc}
A_{11} & A_{12} \\
0 & A_{22}
\end{array}\right]
$$

for nontrivial square matrices $A_{11}, A_{22}$. The spectrum of $A$ is denoted $\Lambda(A)=\left\{\lambda_{1}(A), \ldots, \lambda_{n}(A)\right\}$, where $\lambda_{i}(A), i=$ $1, \ldots, n$, are the eigenvalues of $A$. The spectral radius of $A$, $\rho(A)$, is the smallest positive real number such that $\rho(A) \geqslant$ $\left|\lambda_{i}(A)\right|, \forall i=1, \ldots, n$. The spectral abscissa of $A$ is $\mu(A)=$ $\max \left\{\operatorname{Re}\left(\lambda_{i}(A), i=1, \ldots, n\right\}\right.$.

The following standard properties of nonnegative matrices will be needed later on.

Theorem 1 (Perron-Frobenius). Let $A \in \mathbb{R}^{n \times n} \geqslant 0$ be irreducible. Then $\rho(A)$ is a real, positive, algebraically simple eigenvalue of $A$, of right (resp. left) eigenvector $v>0$ (resp. $w>0)$.

Lemma 1 If $A \geqslant 0$ irreducible, then either

$$
\rho(A)=\sum_{j=1}^{n} a_{i j} \quad \forall i=1, \ldots, n
$$

or

$$
\min _{i}\left(\sum_{j=1}^{n} a_{i j}\right)<\rho(A)<\max _{i}\left(\sum_{j=1}^{n} a_{i j}\right) .
$$

In addition, if $x>0$ and $\lambda \in \mathbb{R}_{+}$then

$$
\begin{aligned}
& A x>\lambda x \quad \Longrightarrow \quad \rho(A)>\lambda \\
& A x<\lambda x \quad \Longrightarrow \quad \rho(A)<\lambda .
\end{aligned}
$$

\section{B. Concave and subhomogeneous vector fields}

Consider a convex set $\mathcal{W} \subset \mathbb{R}^{n}$. A function $f: \mathcal{W} \rightarrow \mathbb{R}^{n}$ is said to be non-decreasing in $\mathcal{W}$ if $x_{1} \leqslant x_{2}$ implies $f\left(x_{1}\right) \leqslant$ $f\left(x_{2}\right) \forall x_{1}, x_{2} \in \mathcal{W}$. It is said to be increasing if in addition $x_{1}<x_{2}$ implies $f\left(x_{1}\right)<f\left(x_{2}\right)$.

Given $\mathcal{W} \subset \mathbb{R}^{n}$ convex, $f: \mathcal{W} \rightarrow \mathbb{R}^{n}$ is said to be concave if

$$
f\left(\alpha x_{1}+(1-\alpha) x_{2}\right) \geqslant \alpha f\left(x_{1}\right)+(1-\alpha) f\left(x_{2}\right)
$$

$\forall x_{1}, x_{2} \in \mathcal{W}$ and $\forall 0 \leqslant \alpha \leqslant 1$. It is said to be strictly concave if the inequality in (3) is strict in $0<\alpha<1 \forall x_{1}, x_{2} \in \mathcal{W}$, $x_{1, i} \neq x_{2, i}, i=1, \ldots, n$. For a concave vector field $f$, the tangent vector must always overestimate $f$ at any point and 
viceversa. Therefore we have that a $C^{1}$ vector field $f: \mathcal{W} \rightarrow$ $\mathbb{R}^{n}$ is concave if and only if

$$
f\left(x_{1}\right) \leqslant f\left(x_{2}\right)+\frac{\partial f\left(x_{2}\right)}{\partial x}\left(x_{1}-x_{2}\right)
$$

$\forall x_{1}, x_{2} \in \mathcal{W} . f$ is strictly concave if (4) holds strictly $\forall x_{1}, x_{2} \in \mathcal{W}, x_{1, i} \neq x_{2, i}, i=1, \ldots, n$. Clearly, $f$ strictly concave and non-decreasing means $f$ increasing.

The vector field $f: \mathcal{W} \rightarrow \mathbb{R}^{n}$ is said to be subhomogeneous of degree $\tau>0$ if

$$
f(\alpha x) \geqslant \alpha^{\tau} f(x)
$$

$\forall x \in \mathcal{W}$ and $0 \leqslant \alpha \leqslant 1$, and strictly subhomogeneous if the inequality (5) holds strictly $\forall x \in \mathcal{W}$ and $0<\alpha<1$.

We will need the following lemma, whose proof can be found in [5].

Lemma 2 The vector field $f: \mathcal{W} \rightarrow \mathbb{R}^{n}$ is subhomogeneous of degree $\tau>0$ if and only if

$$
\frac{\partial f}{\partial x}(x) x \leqslant \tau f(x) \quad \forall x \geqslant 0 .
$$

It is strictly subhomogeneous if and only if the inequality holds strictly $\forall x \geqslant 0$.

Another auxiliary lemma used later is the following.

Lemma 3 If $f: \mathcal{W} \rightarrow \mathbb{R}^{n}$ is subhomogeneous of degree $\tau_{1}$, $0<\tau_{1}<1$, then it is also subhomogeneous of degree $\tau_{2}$, $0<\tau_{1} \leqslant \tau_{2} \leqslant 1$.

Proof. For $\alpha \in(0,1), \tau_{1} \leqslant \tau_{2}$ implies $\alpha^{\tau_{1}} \geqslant \alpha^{\tau_{2}}$, hence in (5)

$$
f(\alpha x) \geqslant \alpha^{\tau_{1}} f(x) \geqslant \alpha^{\tau_{2}} f(x) .
$$

In the case of $f(0) \geqslant 0$, concavity is related to subhomogeneity of degree 1.

Proposition 1 Consider $\mathcal{W} \subset \mathbb{R}^{n}$ convex, $0 \in \mathcal{W}$, and let $f: \mathcal{W} \rightarrow \mathbb{R}^{n}$ be a vector field such that $f(0) \geqslant 0$. If $f(x)$ is concave then $f(x)$ is subhomogeneous of degree 1.

Proof. Choosing $x_{2}=0$ in (3), we have

$$
f\left(\alpha x_{1}\right) \geqslant \alpha f\left(x_{1}\right)+(1-\alpha) f(0),
$$

or $f\left(\alpha x_{1}\right) \geqslant \alpha f\left(x_{1}\right)$, since in $\alpha \in[0,1](1-\alpha) f(0) \geqslant 0$.

\section{Positive and cooperative systems}

Given a system

$$
\dot{x}=h(x), \quad x(0)=x_{o}
$$

let $x\left(t, x_{o}\right)$ denote its forward solution from the initial condition $x_{o}$ (assumed to be defined $\forall t \in[0, \infty)$ ). The system (6) is said to be positive if $x\left(t, x_{o}\right) \in \mathbb{R}_{+}^{n} \forall x_{o} \in \mathbb{R}_{+}^{n}$, i.e., $\mathbb{R}_{+}^{n}$ is forward-invariant for (6). Assuming uniqueness of the solution of (6), it is shown for instance in [1] that a necessary and sufficient condition for positivity is that $x_{i}=0$ implies $h_{i}(x) \geqslant 0 \forall x \in \operatorname{bd}\left(\mathbb{R}_{+}^{n}\right)$.

The system (6) is said to be monotone if $\forall x_{1}, x_{2} \in \mathcal{W}$ with $x_{1} \leqslant x_{2}$ it holds that $x\left(t, x_{1}\right) \leqslant x\left(t, x_{2}\right)$. The Kamke condition gives an easily testable characterization of monotonicity (see [31], par. 3.1 for more details): the vector field $h(x): \mathcal{W} \rightarrow \mathbb{R}^{n}$ is said to be of type-K or to satisfy the Kamke condition if for each $i=1, \ldots, n, h_{i}(a) \leqslant h_{i}(b)$ $\forall a, b \in \mathcal{W}$ satisfying $a \leqslant b$ and $a_{i}=b_{i}$. A type-K system is monotone. For $C^{1}$ vector fields, type-K systems admit an infinitesimal characterization in terms of the signs of the Jacobian. We are particularly interested in a subclass of monotone systems called cooperative systems. A vector field $h: \mathcal{W} \rightarrow \mathbb{R}^{n}$ is said to be cooperative if the Jacobian matrix $H(x)=\frac{\partial h(x)}{\partial x}$ is Metzler $\forall x \in \mathcal{W}$. Similarly, the system (6) is said to be cooperative if the vector field $h$ is cooperative on $\mathcal{W}=\mathbb{R}_{+}^{n} \backslash\{0\}$. From $H(x)$ Metzler, it can be easily shown that if the system (6) is cooperative then $\mathbb{R}_{+}^{n}$ is a forwardinvariant set for it, i.e., the system (6) is a positive system.

An important property of cooperative systems that will be used to prove convergence is given by the following lemma whose proof can be found e.g. in [31] Prop. 3.2.1.

Lemma 4 Let $\mathcal{W}$ be open and $h(x): \mathcal{W} \rightarrow \mathbb{R}^{n}$ be a cooperative vector field. If $\exists x_{o} \in \mathcal{W}$ for which $h\left(x_{o}\right)<0$ (resp. $h\left(x_{o}\right)>0$ ), then the trajectory $x\left(t, x_{o}\right)$ of (6) is decreasing (resp. increasing) for $t \geqslant 0$. In the case $h\left(x_{o}\right) \leqslant 0$ (resp. $h\left(x_{o}\right) \geqslant 0$ ), the trajectory $x\left(t, x_{o}\right)$ of (6) is nonincreasing (resp. non-decreasing).

\section{Existence and uniqueness of positive equilibria}

For a function $f: \mathcal{W} \rightarrow \mathbb{R}^{n}$, we are interested in fixed points of the form $x^{*} \in \mathcal{W}$ such that $f\left(x^{*}\right)=x^{*}$. We want to determine conditions under which $f$ admits a unique such fixed point. For existence, we can use a well-known theorem, valid for non-decreasing functions.

Theorem 2 (Tarski fixed point theorem). Given $\mathcal{W} \subset \mathbb{R}^{n}$ convex, assume $f: \mathcal{W} \rightarrow \mathcal{W}$ is a nondecreasing continuous function such that $f\left(x_{1}\right)>x_{1}$ for some $x_{1} \in \mathcal{W}, x_{1}>0$ and $f\left(x_{2}\right)<x_{2}$ for some $x_{2} \in \mathcal{W}, x_{2}>x_{1}$. Then $\exists x^{*} \in \mathcal{W}$ such that $f\left(x^{*}\right)=x^{*}$.

Proof. See [20].

In the following we shall focus on the case of $\mathcal{W}=\mathbb{R}_{+}^{n}$. Under some extra condition like concavity, $f$ can be shown to have a unique fixed point, see [20]. The following theorem generalizes the uniqueness result of Kennan to subhomogeneous vector fields. Its proof is a slight variation of the one proposed in [20].

Theorem 3 Let $f: \mathbb{R}_{+}^{n} \rightarrow \mathbb{R}_{+}^{n}$ be continuous and such that

1) $f$ is strictly subhomogeneous of degree $\tau>0$,

2) $f$ is increasing,

3) $f(0) \geqslant 0$,

4) $\exists x_{1} \in \operatorname{int}\left(\mathbb{R}_{+}^{n}\right)$ such that $f\left(x_{1}\right)>x_{1}$, 
5) $\exists x_{2} \in \operatorname{int}\left(\mathbb{R}_{+}^{n}\right), x_{2}>x_{1}$, such that $f\left(x_{2}\right)<x_{2}$, then $\exists$ unique $x^{*} \in \operatorname{int}\left(R_{+}^{n}\right)$ such that $x^{*}=f\left(x^{*}\right)$.

Proof. See Appendix A.

From Proposition 1 , if $f$ is strictly concave and $f(0) \geqslant 0$ the previous theorem holds as a special case (corresponding to $\tau=1$ ).

\section{CONCAVE SYSTEMS: EQUILIBRIA AND STABILITY}

The class of nonlinear positive systems considered in this paper is the following:

$$
\dot{x}=\Delta(-x+f(x)),
$$

where $x \in \mathbb{R}_{+}^{n}, f: \mathbb{R}_{+}^{n} \rightarrow \mathbb{R}_{+}^{n}$ is a $C^{1}$ cooperative vector field such that $F(x)=\frac{\partial f(x)}{\partial x} \geqslant 0 \forall x \in \mathbb{R}_{+}^{n}$, and $\Delta=\operatorname{diag}\left(\delta_{1}, \ldots, \delta_{n}\right), \delta_{i}>0$. The presence of a negative diagonal term in (7) implies that the complete Jacobian of (7) is Metzler, hence (7) is a cooperative system. Since $\Delta$ is invertible and $F(x)$ can have nonzero diagonal entries, the form (7) covers most cooperative systems used in the literature (exceptions are those having nonlinear negative diagonal terms in $F(x)$ ).

\section{A. A spectral characterization of existence and uniqueness of equilibria}

The following theorem gives a spectral condition for the existence and uniqueness of a positive fixed point, in the case of concave and increasing vector fields.

Theorem 4 Consider the system (7), with $f: \mathbb{R}_{+}^{n} \rightarrow \mathbb{R}_{+}^{n}$ a $C^{1}$, strictly concave and increasing vector field, $f(0)=0$. Assume $F(x) \geqslant 0$ and irreducible $\forall x \in \mathbb{R}_{+}^{n}$. If the following conditions hold

1) $\rho(F(0))>1$;

2) $\exists x_{2} \in \operatorname{int}\left(\mathbb{R}_{+}^{n}\right)$ such that $\rho\left(F\left(x_{2}\right)\right)<1$,

then the system admits a unique positive equilibrium $x^{*} \in$ $\operatorname{int}\left(\mathbb{R}_{+}^{n}\right)$.

Proof. See Appendix B.

Clearly, since $f(0)=0$, the origin is also an equilibrium point of (7), distinct from $x^{*}$.

Remark 1 While the first condition of Theorem 4 can be found in papers such as [32], the second does not seem to appear explicitly in the literature.

The value of the spectral radius at the equilibrium point is a result of independent interest ${ }^{1}$.

Proposition 2 Let $f: \mathbb{R}_{+}^{n} \rightarrow \mathbb{R}_{+}^{n}$ be a strictly concave and increasing vector field, $f(0)=0$. Assume $F(x) \geqslant 0$ and irreducible $\forall x \in \mathbb{R}_{+}^{n}$. If $\exists x^{*} \in \operatorname{int}\left(\mathbb{R}_{+}^{n}\right)$ such that $f\left(x^{*}\right)=$ $x^{*}$, then $\rho\left(F\left(x^{*}\right)\right)<1$.

\footnotetext{
${ }^{1}$ It explains the asymmetry in the proof of the second part of the auxiliary Lemma 5: in one direction $\bar{x}<x_{2}$, in the other $\bar{x}=x_{2}$, see Appendix B.
}

Proof. See Appendix B.

Another related result of interest is the following:

Proposition 3 Let $f: \mathbb{R}_{+}^{n} \rightarrow \mathbb{R}_{+}^{n}$ be $C^{1}$ strictly concave and increasing. If $\exists x_{2} \in \operatorname{int}\left(\mathbb{R}_{+}^{n}\right)$ such that $f\left(x_{2}\right)<x_{2}$, then $f(x)<x \forall x>x_{2}$.

Proof. See Appendix B.

If in addition to strict concavity and monotonicity we also add a boundedness assumption on $f$, then the spectral radius must decrease to zero when $\|x\|$ grows.

Proposition 4 Let $f: \mathbb{R}_{+}^{n} \rightarrow[0, q]^{n} \subset \mathbb{R}_{+}^{n}$ be $C^{1}$ strictly concave, increasing and bounded, $q>0$. Assume $F(x) \geqslant 0$ and irreducible $\forall x \in \mathbb{R}_{+}^{n}$. Then $\lim _{\|x\| \rightarrow \infty} \rho(F(x))=0$.

Proof. See Appendix B.

\section{B. A spectral characterization of stability}

As for its existence and uniqueness, the stability properties of a strictly positive equilibrium can be determined by checking a spectral condition on the Jacobian.

Theorem 5 Consider the system (7), with $f: \mathbb{R}_{+}^{n} \rightarrow \mathbb{R}_{+}^{n}$ a $C^{1}$, strictly concave and increasing vector field, $f(0)=0$. Assume $F(x) \geqslant 0$ and irreducible $\forall x \in \mathbb{R}_{+}^{n}$.

1) If $\rho(F(0))<1$ then the origin is an asymptotically stable equilibrium point for (7), with domain of attraction $\mathcal{A}(0)$ which contains $\mathbb{R}_{+}^{n}$.

2) If $\rho(F(0))>1$ and $\exists x_{2} \in \operatorname{int}\left(\mathbb{R}_{+}^{n}\right)$ such that $\rho\left(F\left(x_{2}\right)\right)<1$, the unique positive equilibrium $x^{*} \in$ $\operatorname{int}\left(\mathbb{R}_{+}^{n}\right)$ of system (7) is asymptotically stable and has domain of attraction $\mathcal{A}\left(x^{*}\right) \supset \mathbb{R}_{+}^{n} \backslash\{0\}$.

Proof. See Appendix C.

The conditions of Theorem 5 are sufficiently simple to check, in particular when $f$ is also bounded, as the existence of $x_{2}$ is automatically guaranteed in that case (Proposition 4).

Remark 2 When $x^{*}>0$ exists, then it follows from Proposition 2 that $\rho\left(F\left(x^{*}\right)\right)<1$. This condition can be used to show that the symmetric part of the Jacobian of (7) is negative definite around $x^{*}$. The condition is however only local, and it is lost near 0 , where $\rho(F(0))>1$. Hence the contraction analysis approach of [25] cannot be used for these systems. The system is contracting only in the Hilbert projective norm [23], not in the Riemannian/Euclidean norm used in [25].

\section{GENERALIZATIONS OF THE SPECTRAL CONDITIONS}

In this Section, the results of Theorem 4 and Theorem 5 are extended to two other classes of vector fields: subhomogeneous and contractive. 


\section{A. Subhomogeneous vector fields}

We will treat only the case of positive equilibrium point. The stability condition for the origin is in fact analogous to condition 1 of Theorem 5 and is not repeated here (see also [5]).

Theorem 6 Consider the system (7) with $f: \mathbb{R}_{+}^{n} \rightarrow \mathbb{R}_{+}^{n} a$ $C^{1}$ vector field which is strictly subhomogeneous of degree $0<\tau \leqslant 1$ and increasing. Assume $f(0)=0$ and $F(x) \geqslant 0$ irreducible $\forall x \in \mathbb{R}_{+}^{n}$. If the following conditions hold:

1) $\rho(F(0))>1$;

2) $\exists x_{2} \in \operatorname{int}\left(\mathbb{R}_{+}^{n}\right)$ such that $\rho\left(F\left(x_{2}\right)\right) \leqslant \zeta<1$ and $\rho(F(x)) \leqslant \zeta \forall x>x_{2}$,

then the system (7) admits a unique positive equilibrium point $x^{*} \in \operatorname{int}\left(\mathbb{R}_{+}^{n}\right)$ which is asymptotically stable with domain of attraction $\mathcal{A}\left(x^{*}\right) \supset \mathbb{R}_{+}^{n} \backslash\{0\}$.

Proof. See Appendix D.

Notice that Theorem 6 can be extended to $f$ which are monotone and subhomogeneous but increasing only in a rectangular subset of $\mathbb{R}_{+}^{n}$ (replacing $F(x)$ nonnegative with $F(x)$ Metzler).

\section{B. Contractive vector fields}

Definition 1 A function $\phi: \mathbb{R}_{+}^{n} \rightarrow \mathbb{R}_{+}^{n}$ is said to be a ccontractive interference function if the following properties are satisfied $\forall x \in \mathbb{R}_{+}^{n}$ :

1) $\phi(x)>0$;

2) if $x_{1} \leqslant x_{2}$, then $\phi\left(x_{1}\right) \leqslant \phi\left(x_{2}\right)$;

3) $\exists$ a constant $c \in[0,1)$ and a vector $\nu>0$ such that $\forall \epsilon>0$

$$
\phi(x+\epsilon \nu) \leqslant \phi(x)+c \epsilon \nu .
$$

If we consider a system like (7) with $f(x)=\phi(x)$, then contractive interference functions guarantee existence, uniqueness and asymptotic stability of a positive equilibrium point [10]. Clearly, condition 1 of Definition 1 implies that the origin can never be an equilibrium point for (7). The following proposition characterizes (differentiable) contractive interference functions in terms of the spectrum of their Jacobian $\Phi(x)=\frac{\partial \phi(x)}{\partial x}$.

Proposition 5 If $\phi(x)$ is a $C^{1}$ c-contractive interference function, then

$$
\rho(\Phi(x))<1 \quad \forall x \in \operatorname{int}\left(\mathbb{R}_{+}^{n}\right) .
$$

Proof. See Appendix E.

For increasing functions whose Jacobian is irreducible, a converse result is also true.

Proposition 6 If $\phi(x)$ is a $C^{1}$ increasing function such that the following hold $\forall x \in \mathbb{R}_{+}^{n}$ :

1) $\phi(x)>0$,

2) $\rho(\Phi(x)) \leqslant \zeta_{0}<1$,

3) $\Phi(x)$ irreducible, then $\phi(x)$ is a c-contractive interference function.

Proof. See Appendix F.

Remark 3 The infinitesimal spectral characterization given in Propositions 5 and 6 extends to the nonlinear case a wellknown property of the spectral radius of linear interference functions, see [10], [28], [35].

The known properties of c-contractive interference functions imply that we have the following spectral characterization for existence, uniqueness and stability of a positive equilibrium point.

Theorem 7 Consider the system (7) with $f: \mathbb{R}_{+}^{n} \rightarrow \mathbb{R}_{+}^{n} a$ $C^{1}$ increasing vector field. Assume $f(x)>0 \forall x \in \mathbb{R}_{+}^{n}$ and $F(x)$ irreducible $\forall x \in \mathbb{R}_{+}^{n}$. If $\rho(F(x))<1 \forall x \in \operatorname{int}\left(\mathbb{R}_{+}^{n}\right)$, the system (7) admits a unique positive equilibrium point $x^{*} \in \operatorname{int}\left(\mathbb{R}_{+}^{n}\right)$ which is asymptotically stable with domain of attraction $\mathcal{A}\left(x^{*}\right) \supset \mathbb{R}_{+}^{n}$.

Proof. See Appendix G.

It is worth noticing that the class of concave-increasing positive vector fields and that of c-contractive vector fields are non-identical, although both may give rise to a single positive attractor. For instance, it follows from Theorem 5 that in order to admit a positive equilibrium point in a neighbourhood of the origin, a concave-increasing function must have a spectral radius $>1$, while $\rho(F(x))<1$ always for a c-contractive function. On the other hand, the following system, taken from [10], is an example of a non-concave c-contractive $C^{1}$ scalar function:

$$
f(x)= \begin{cases}x^{2}+\frac{1}{100} & 0 \leqslant x \leqslant \frac{1}{4} \\ \frac{x}{2}-\frac{1}{16}+\frac{1}{100} & x>\frac{1}{4} .\end{cases}
$$

Clearly, for c-contractive vector fields $f(0)>0$ is a nondispensable assumption.

Remark 4 The condition $\rho(F(x))<1 \forall x \in \mathbb{R}_{+}^{n}$ implies that the symmetric part of the Jacobian of (7) is always negative definite. Hence, unlike for the concave/subhomogeneous vector fields passing through the origin, convergence of ccontractive vector fields can be shown also using the contraction analysis approach of [25].

\section{SPECTRAL CONDITIONS FOR STABILIZABILITY}

In this Section we use the results of Section III to determine linear diagonal state feedback laws for the stabilization to a positive equilibrium of the control system

$$
\dot{x}=\tilde{f}(x)+u,
$$

where $\tilde{f}(x)$ is cooperative and concave/subhomogeneous, and $u=\left[\begin{array}{lll}u_{1} & \ldots & u_{n}\end{array}\right]^{T}$ is a vector of control inputs. The 
simplest possible solution is to make use of a linear diagonal state feedback

$$
u=-K x=-\left[\begin{array}{lll}
k_{1} & & \\
& \ddots & \\
& & k_{n}
\end{array}\right] x .
$$

Our task in the following is to determine conditions on $K$ that guarantee the stabilizability of the closed loop system (9)-(10).

Theorem 8 Consider the system (9), with $\tilde{f}: \mathbb{R}_{+}^{n} \rightarrow \mathbb{R}_{+}^{n} a$ $C^{1}$, strictly concave and increasing vector field, $\tilde{f}(0)=0$. Assume $\tilde{F}(x)=\frac{\partial \tilde{f}(x)}{\partial x} \geqslant 0$ irreducible $\forall x \in \mathbb{R}_{+}^{n}$. Consider the feedback law (10).

1) If $K$ such that $k_{\min }=\min _{i}\left(k_{i}\right)>\rho(\tilde{F}(0))$, then the origin is an asymptotically stable equilibrium point for the closed loop system (9)-(10). In this case the domain of attraction $\mathcal{A}(0)$ contains $\mathbb{R}_{+}^{n}$.

2) If $K$ is such that $k_{\max }=\max _{i}\left(k_{i}\right)<\rho(\tilde{F}(0))$ and $k_{\min }>\rho\left(\tilde{F}\left(x_{2}\right)\right)$ for some $x_{2} \in \operatorname{int}\left(\mathbb{R}_{+}^{n}\right)$, then the closed-loop system (9)-(10) admits a unique positive equilibrium $x^{*} \in \operatorname{int}\left(\mathbb{R}_{+}^{n}\right)$ which is asymptotically stable and has domain of attraction $\mathcal{A}\left(x^{*}\right) \supset \mathbb{R}_{+}^{n} \backslash\{0\}$.

Proof. See Appendix H.

The following is a straightforward combination of Theorem 8 and Theorem 6.

Corollary 1 Theorem 8 holds unchanged if we replace "strictly concave" with "strictly subhomogeneous".

When as in Section IV-B $\tilde{f}(0)>0$, then the feedback design simplifies considerably (the origin is no longer an equilibrium point). The following corollary follows.

Corollary 2 Consider the system (9) with $\tilde{f}: \mathbb{R}_{+}^{n} \rightarrow \mathbb{R}_{+}^{n}$ a $C^{1}$ increasing vector field. Assume $\tilde{f}(x)>0 \forall x \in \mathbb{R}_{+}^{n}$ and $\tilde{F}(x)$ irreducible $\forall x \in \mathbb{R}_{+}^{n}$. Consider the feedback law (10). If $K$ such that $k_{\min }>\rho(\tilde{F}(x)) \forall x \in \operatorname{int}\left(\mathbb{R}_{+}^{n}\right)$, then the closed-loop system (9)-(10) admits a unique positive equilibrium point $x^{*} \in \operatorname{int}\left(\mathbb{R}_{+}^{n}\right)$ which is asymptotically stable with domain of attraction $\mathcal{A}\left(x^{*}\right) \supset \mathbb{R}_{+}^{n}$.

Proof. See Appendix H.

\section{APPLICATION TO INTERCONNECTED SYSTEMS}

In this section we consider an interconnected system on a given graph $\mathcal{G}$, i.e., a system in which the state of a node propagates to its first neighbours following the direction of the edges. The incoming interactions at a node obey a principle of linear superposition of the effects.

\section{A. Stability analysis for concave interconnections}

Assume the network dynamics includes first order degradation terms $\delta_{i}, i=1, \ldots, n$, on the diagonal. Assume further that a node $j$ exerts the same form of influence on all its neighbours, up to a scaling constant which corresponds to the weight of the edge connecting $j$ with $i$. If $A=\left[a_{i j}\right] \geqslant 0$ is the weighted adjacency matrix of the network, and $\psi_{j}\left(x_{j}\right)$ : $\mathbb{R}_{+} \rightarrow \mathbb{R}$ is the functional form of the interaction from node $j$ to all its neighbours, then we can write the system as

$$
\frac{d x}{d t}=A \psi(x)-\Delta x
$$

where $\psi(x)=\left[\psi_{1}\left(x_{1}\right) \ldots \psi_{n}\left(x_{n}\right)\right]^{T}$. We assume that each $\psi_{j}\left(x_{j}\right)$ is increasing and strictly concave. Additionally, we enforce a boundedness condition on $\psi_{j}$ :

$$
\lim _{x_{j} \rightarrow+\infty} \psi_{j}\left(x_{j}\right)=1 \text {. }
$$

While not necessary for the application of Theorem 4 and Theorem 5, from Proposition 4, the condition (12) implies that the existence of $x_{2}>0$ such that $\rho\left(F\left(x_{2}\right)\right)<1$ in these two theorems is automatically satisfied.

The system (11) can be rewritten in the form (7) if we denote $f(x)=\Delta^{-1} A \psi(x)$. Its Jacobian is then

$$
\begin{aligned}
F(x)=\Delta^{-1} \tilde{F}(x) & =\Delta^{-1} A \frac{\partial \psi(x)}{\partial x} \\
& =\Delta^{-1} A\left[\begin{array}{ccc}
\frac{\partial \psi_{1}\left(x_{1}\right)}{\partial x_{1}} & & \\
& \ddots & \\
& & \frac{\partial \psi_{n}\left(x_{n}\right)}{\partial x_{n}}
\end{array}\right] .
\end{aligned}
$$

From (20) and $A \geqslant 0$, it follows that $F(x) \geqslant 0 \forall x \in \mathbb{R}_{+}^{n}$. This implies that (11) is a positive cooperative system.

Calling $\mathcal{G}(F(x))$ the graph whose adjacency matrix is $F(x), F(x)$ and $A$ have the same graph at each point of $\mathbb{R}_{+}^{n}$, hence irreducibility of $A$ implies irreducibility of $F(x)$ $\forall x \in \operatorname{int}\left(\mathbb{R}_{+}^{n}\right)$. Since $\psi_{j}\left(x_{j}\right)$ is strictly concave, so is $f(x)$. Hence Theorems 4 and 5 are applicable. Furthermore, for interconnected systems we have the following monotonicity condition on the spectral radius.

Proposition 7 Let $\psi_{i}: \mathbb{R}_{+} \rightarrow \mathbb{R}, i=1, \ldots, n$, be $C^{1}$ strictly concave, non-decreasing, and such that $\psi_{i}(0)=0$. If $A \geqslant 0$ irreducible, then

$$
\rho\left(F\left(x_{1}\right)\right)>\rho\left(F\left(x_{2}\right)\right) \quad \forall x_{1}, x_{2} \in \mathbb{R}_{+}^{n}, x_{1}<x_{2} .
$$

Proof. See Appendix I.

An obvious corollary is the following.

Corollary 3 Under the same hypothesis as Proposition 7,

$$
\rho(F(0))>\rho(F(x)) \quad \forall x \in \operatorname{int}\left(\mathbb{R}_{+}^{n}\right) .
$$

A special case of (11) is the following distributed dynamics, adapted from the bio-inspired collective decision-making system of [12]:

$$
\dot{x}_{i}=-\delta_{i} x_{i}+\pi \sum_{j=1}^{n} a_{i j} \psi_{j}\left(x_{j}\right), \quad i=1, \ldots, n,
$$


where $\pi \in[0,+\infty)$ is a scalar parameter, $A \geqslant 0$ off-diagonal such that $\delta_{i}=\sum_{j=1}^{n} a_{i j}, i=1, \ldots, n$ (i.e., $\Delta-A$ is Laplacian matrix), and $\psi_{j}: \mathbb{R}_{+} \rightarrow[0,1), j=1, \ldots, n$, are smooth functions that satisfy the following conditions:

$$
\begin{array}{ll}
\mathrm{H} 1: & \psi_{j}(0)=0 ; \\
\mathrm{H} 2: & \frac{\partial \psi_{j}}{\partial x_{j}}\left(x_{j}\right)>0, \frac{\partial \psi_{j}}{\partial x_{j}}(0)=1 \text { and } \lim _{x_{j} \rightarrow+\infty} \frac{\partial \psi_{j}}{\partial x_{j}}\left(x_{j}\right)=0 ; \\
\mathrm{H} 3: & \psi_{j} \text { strictly concave. }
\end{array}
$$

In [12], the system (15) (with slightly different hypothesis on $\psi$ ) is studied for $x \in \mathbb{R}^{n}$, while here we are interested only in the positive orthant. In order to describe (locally) the behavior of (15) as $\pi$ varies, [12] makes use of bifurcation theory. The following proposition shows that for any value of $\pi$ it can be described efficiently (and globally in $\mathbb{R}_{+}^{n}$ ) also by using the tools developed in this paper. If we rewrite (15) as

$$
\dot{x}=\Delta(x-\pi \hat{A} \psi(x))
$$

where $\hat{A}=\Delta^{-1} A$, then it is evident that (16) is in the form (7). From condition H2, in the origin $\frac{\partial \psi}{\partial x}(0)=I$, hence $F(0)=\hat{A}=\Delta^{-1} A$.

Proposition 8 Consider the system (15) with $A \geqslant 0$ irreducible and $\psi$ obeying $\mathrm{HI}-\mathrm{H} 3$.

1) If $\pi<1$ the origin is an asymptotically stable equilibrium point, with domain of attraction $\mathcal{A}(0) \subset \mathbb{R}_{+}^{n}$.

2) If $\pi>1$ then the unique positive equilibrium point $x^{*} \in \operatorname{int}\left(\mathbb{R}_{+}^{n}\right)$ is asymptotically stable with domain of attraction $\mathcal{A}\left(x^{*}\right) \subset \mathbb{R}_{+}^{n} \backslash\{0\}$.

Proof. See Appendix J.

\section{$B$. Interconnected systems of c-contractive interference func- tions}

For interconnected systems, c-contractive interference functions can be obtained from concave functions by shifting them away from the origin.

Proposition 9 Let $\psi_{i}: \mathbb{R}_{+} \rightarrow \mathbb{R}, i=1, \ldots, n$, be $C^{1}$ strictly concave, non-decreasing, and such that $\psi_{i}(0)=0$. Assume $A \geqslant 0$ irreducible, and consider

$$
\phi(x)=A \psi(x)+p, \quad p>0 .
$$

If $\rho(\Phi(0))<1$ then $\phi(x)$ is a c-contractive interference function.

\section{Proof. See Appendix K.}

Consequently, Theorem 7 holds for our concave $\psi$, as stated in the following corollary.

Corollary 4 Let $\psi_{i}: \mathbb{R}_{+} \rightarrow \mathbb{R}, i=1, \ldots, n$, be $C^{1}$ strictly concave, non-decreasing, and such that $\psi_{i}(0)=0$. Assume $A \geqslant 0$ irreducible. Consider the interference functions (17). If $\rho(\Phi(0))<1$, then the system (7) with $f(x)=\phi(x)$ admits a unique positive equilibrium point $x^{*} \in \operatorname{int}\left(\mathbb{R}_{+}^{n}\right)$ which is asymptotically stable with domain of attraction $\mathcal{A}\left(x^{*}\right) \supset \mathbb{R}_{+}^{n}$.

\section{Distributed feedback stabilization}

If instead of the autonomous system (15), we have as in Section V the control system

$$
\dot{x}_{i}=\sum_{j=1}^{n} a_{i j} \psi_{j}\left(x_{j}\right)+u_{i}, \quad i=1, \ldots, n,
$$

with $u_{i}$ control inputs, then the task becomes to design a state feedback law for (18) so that stability is imposed on either the origin or a strictly positive equilibrium point. Theorem 8 can be used for this scope. However, in the context of interconnected systems, a limitation of Theorem 8 is that it requires the knowledge of the spectral radius of the interaction part. Hence such control laws cannot be implemented in a distributed fashion, i.e., with only the knowledge of the state of the neighbours of a node according to $\mathcal{G}(A)$. The following proposition determines linear diagonal feedback laws that make use of only such information.

Proposition 10 Consider the system (18) with $A \geqslant 0$ irreducible and $\psi$ obeying $\mathrm{HI}-\mathrm{H} 3$. Consider the feedback law (10).

1) If $K$ such that $k_{i}>\sum_{j} a_{i j}, i=1, \ldots, n$, then the origin is an asymptotically stable equilibrium point, with domain of attraction $\mathcal{A}(0) \subset \mathbb{R}_{+}^{n}$.

2) If $K$ such that $k_{i}<\sum_{j} a_{i j}, i=1, \ldots, n$, then the unique positive equilibrium point $x^{*} \in \operatorname{int}\left(\mathbb{R}_{+}^{n}\right)$ is asymptotically stable with domain of attraction $\mathcal{A}\left(x^{*}\right) \subset \mathbb{R}_{+}^{n} \backslash\{0\}$.

Proof. See Appendix L.

\section{Examples}

The system (11) with the extra condition (12) resembles closely a cooperative additive neural network of Hopfield type but lacks external inputs. Such neural networks models are sometimes referred to as (cooperative) Cohen-Grossberg neural networks [37]. An example of $\psi_{j}\left(x_{j}\right)$ monotone, strictly concave and saturating is given by a so-called Boltzmann sigmoid (or shifted logistic) [14]

$$
\psi_{j}\left(x_{j}\right)=\frac{1-e^{-\frac{x_{j}}{\theta_{j}}}}{1+e^{-\frac{x_{j}}{\theta_{j}}}}
$$

where $\theta_{i}>0$. For (19), $0 \leqslant \psi_{j}\left(x_{j}\right) \leqslant 1$ when $x_{j} \geqslant 0$, and

$$
\frac{\partial \psi_{j}}{\partial x_{j}}=\frac{1}{2 \theta_{j}}\left(1+\psi_{j}\left(x_{j}\right)\right)\left(1-\psi_{j}\left(x_{j}\right)\right)>0 \quad \forall x_{j} \geqslant 0 .
$$

Since $\psi_{j}(0)=0$, the Jacobian linearization at the origin is $1 /\left(2 \theta_{j}\right)$. In particular, when $x_{j} \ll \theta_{j}$ then $\psi_{j}\left(x_{j}\right) \simeq x_{j} / \theta_{j}$ is a first order rate law, while when $x_{j} \gg \theta_{j}$ then $\psi_{i j}\left(x_{j}\right) \simeq 1$ behaves like a zero order rate law. Other monotone concave nonlinearities can be used in place of (19). Many can be found in the neural network literature [14], [19]. Nonlinearities like those in (19) will be considered in the following illustrative examples. 
Example 1 (Interconnected concave system in dim 2) For $n=2$ agents, assuming for example

$$
A=\left[\begin{array}{ll}
0 & 1 \\
1 & 0
\end{array}\right]
$$

the system (11) with the functions (19) becomes

$$
\left\{\begin{array}{l}
\frac{d x_{1}}{d t}=\frac{1-e^{-\frac{x_{2}}{\theta_{2}}}}{1+e^{-\frac{x_{2}}{\theta_{2}}}}-\delta_{1} x_{1} \\
\frac{d x_{2}}{d t}=\frac{1-e^{-\frac{x_{1}}{\theta_{1}}}}{1+e^{-\frac{x_{1}}{\theta_{1}}}}-\delta_{2} x_{2} .
\end{array}\right.
$$

In this case, it is possible to use phase plane analysis to verify the conditions of Theorems 4 and 5 analytically. The nullclines of this system are given by

$$
\left\{\begin{array}{l}
x_{1, \text { null }}=\frac{1-e^{-\frac{x_{2}}{\theta_{2}}}}{\delta_{1}\left(1+e^{-\frac{x_{2}}{\theta_{2}}}\right)} \\
x_{2, \text { null }}=\frac{1-e^{-\frac{x_{1}}{\theta_{1}}}}{\delta_{2}\left(1+e^{-\frac{x_{1}}{\theta_{1}}}\right)}
\end{array}\right.
$$

which for positive $\delta_{i}$ and $\theta_{i}$ have at most 2 intersections in $\mathbb{R}_{+}^{2}$, see Fig. 1. If we look at the graphs of (22), then the slopes at $x=0$ are given by the lines

$$
\left\{\begin{array}{l}
x_{2}=2 \theta_{2} \delta_{1} x_{1} \\
x_{2}=\frac{1}{2 \theta_{1} \delta_{2}} x_{1} .
\end{array}\right.
$$

We have therefore a bifurcation at $\delta_{1} \delta_{2}=\frac{1}{4 \theta_{1} \theta_{2}}$ :

- when $\delta_{1} \delta_{2}>\frac{1}{4 \theta_{1} \theta_{2}}$ the $x_{1}$-nullcline and the $x_{2}$-nullcline intersect only in one equilibrium $\left(x_{0}^{*}=0\right)$;

- when $\delta_{1} \delta_{2}<\frac{1}{4 \theta_{1} \theta_{2}}$ the $x_{1}$-nullcline and the $x_{2}$-nullcline intersect in 2 equilibria: $x_{0}^{*}=0, x_{1}^{*}>0$.

See Fig. 1 for an example.
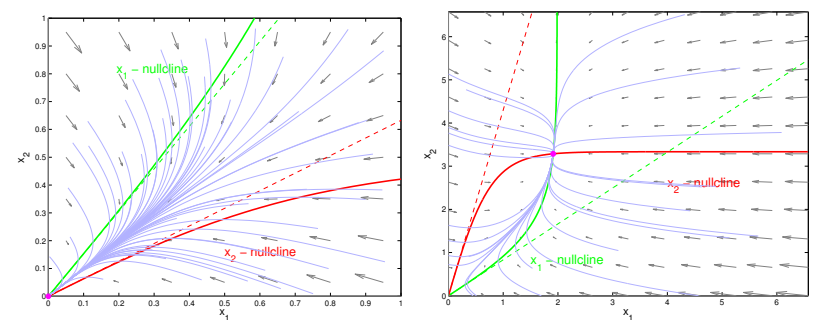

Fig. 1. Example 1. Nullclines are shown as solid lines, slopes at $x=0$ are shown as dashed lines, and a few trajectories are shown in blue solid lines. Left panel: $x_{0}^{*}=0$ is the only equilibrium point. Right panel: $x_{0}^{*}=0$ (unstable) and $x_{1}^{*}>0$ (asymptotically stable) are the two equilibrium points.

The Jacobian of the interaction part alone (omitting the argument in $\psi_{i}$ )

$$
\tilde{F}(x)=\left[\begin{array}{cc}
0 & \frac{\left(1+\psi_{2}\right)\left(1-\psi_{2}\right)}{2 \theta_{2}} \\
\frac{\left(1+\psi_{1}\right)\left(1-\psi_{1}\right)}{2 \theta_{1}} & 0
\end{array}\right]
$$

has eigenvalues

$$
\lambda_{1,2}^{\text {interact. }}= \pm \sqrt{\frac{\left(1+\psi_{1}\right)\left(1-\psi_{1}\right)\left(1+\psi_{2}\right)\left(1-\psi_{2}\right)}{4 \theta_{1} \theta_{2}}},
$$

which implies that the spectral radius of the interaction part is

$$
\rho(\tilde{F}(x))=\sqrt{\frac{\left(1+\psi_{1}\right)\left(1-\psi_{1}\right)\left(1+\psi_{2}\right)\left(1-\psi_{2}\right)}{4 \theta_{1} \theta_{2}}}
$$

from which (as in Proposition 7 and Corollary 3)

$$
\rho(\tilde{F}(0))>\rho(\tilde{F}(x)) \quad \forall x \neq 0,
$$

see Fig. 2. The Jacobian of the entire system (21) is $\tilde{F}(x)-\Delta$ and its eigenvalues are solutions of

$$
\lambda_{1,2}=\frac{\left(\delta_{1}+\delta_{2}\right) \pm \sqrt{\left(\delta_{1}-\delta_{2}\right)^{2}+\frac{\left(1+\psi_{1}\right)\left(1-\psi_{1}\right)\left(1+\psi_{2}\right)\left(1-\psi_{2}\right)}{\theta_{1} \theta_{2}}}}{2} .
$$

Considering an equilibrium point of (21), the conditions for

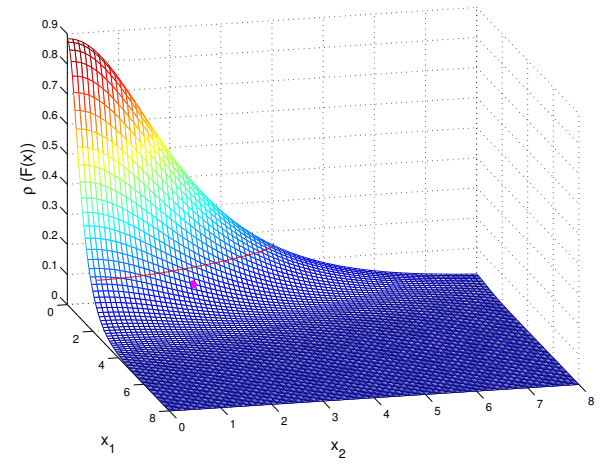

Fig. 2. Example 1. The spectral radius $\rho(\tilde{F}(x))$ is nonnegative and decreasing with $x$. The red contour represents the bifurcation curve $\delta_{1} \delta_{2}=\frac{1}{4 \theta_{1} \theta_{2}}$. The magenta dot represents $x_{1}^{*}$.

its stability are

$$
\begin{aligned}
\operatorname{tr}(\tilde{F}(x)-\Delta) & =-\left(\delta_{1}+\delta_{2}\right)<0 \\
\operatorname{det}(\tilde{F}(x)-\Delta) & =\delta_{1} \delta_{2}-\rho^{2}(\tilde{F}(x))>0 .
\end{aligned}
$$

For example in $x_{0}^{*}=0$, the second condition becomes

$$
\delta_{1} \delta_{2}>\rho^{2}(\tilde{F}(0))=\frac{1}{4 \theta_{1} \theta_{2}}
$$

i.e., when $x_{0}^{*}=0$ is the only equilibrium point of (21) then it must be asymptotically stable. When instead $\delta_{1} \delta_{2}<\frac{1}{4 \theta_{1} \theta_{2}}$ then $x_{0}^{*}$ becomes a saddle point. Since $\left(1+\psi_{i}\right)\left(1-\psi_{i}\right)$ is monotonically decreasing with $x_{i}$, so is $\rho(\tilde{F}(x))$ as a function of $x$, see Fig. 2, and in particular $\lim _{\|x\| \rightarrow \infty} \rho(\tilde{F}(x))=0$. Hence when $\delta_{1} \delta_{2}<\frac{1}{4 \theta_{1} \theta_{2}}=\rho^{2}(\tilde{F}(0))$ it must be $\delta_{1} \delta_{2}>$ $\rho^{2}(\tilde{F}(x))$ for $x$ sufficiently large. In particular this must happen on $x_{1}^{*}$, i.e., the positive equilibrium point of (21) must be asymptotically stable whenever it exists. In conclusion, the system (21) experiences a saddle-node bifurcation at $\delta_{1} \delta_{2}=\rho^{2}(\tilde{F}(0))$. If $\Delta$ is given, only the spectral radius at 0 is needed to discriminate between the two situations described in Theorem 5.

Example 2 (Interconnected concave system in $\operatorname{dim} n$ ) Consider the system (15) with as $\psi_{j}\left(x_{j}\right)$ the Boltzmann sigmoid (19) (suitably normalized so that $\frac{\partial \psi_{j}}{\partial x_{j}}(0)=1$ ). For this system $H 1-H 3$ hold, and the two different behaviors predicted by Proposition 8 can be observed in simulations, 
see Fig. 3. As the parameter $\pi$ passes from $\pi<1$ to $\pi>1$, the system (15) experiences the same saddle-node bifurcation seen in Example 1, i.e., the origin becomes an unstable equilibrium point and a new stable equilibrium point $x^{*}>0$ is created (a global attractor in $\mathbb{R}_{+}^{n}$ ). As shown in the proof of Proposition 8 (see Appendix $\mathrm{J}$ ), at the bifurcation point $\pi=1$ the linearization of (15) is a Laplacian system. Since this is only marginally stable, it cannot predict the stability character of the original nonlinear system. It follows from the strict concavity of $\psi(x)$ that, at $\pi=1, x^{*}=0$ is still an asymptotically stable equilibrium point. To prove it, the Lyapunov argument given in the proof of Theorem 5 (see Appendix C) can be used.
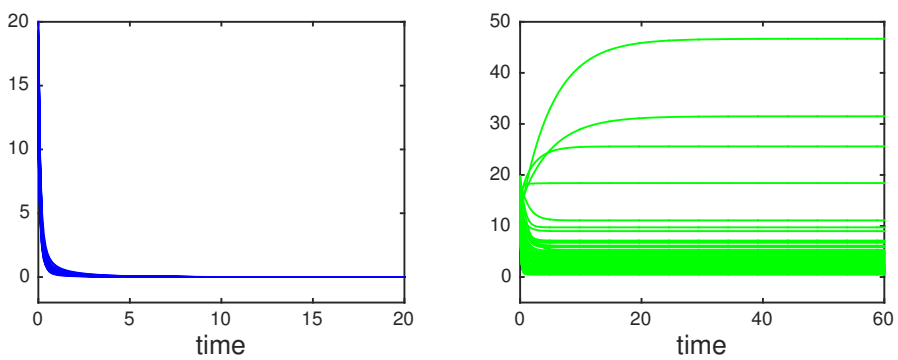

Fig. 3. Example 2. Simulation for a system (15) of $n=100$ nodes, using the functional forms (19) for $\psi$. Left: when $\pi<1$, the origin is asymptotically stable (case 1 of Proposition 8). Right: when $\pi>1, x^{*}>0$ is asymptotically stable (case 2 of Proposition 8).

\section{Example 3 (Interconnected c-contractive system)}

Consider the system (7) with $f(x)=\phi(x)$ the c-contractive interference function given in (17). When the functional forms (19) are used, if $\theta_{i} \geqslant 1 / 2 \forall i=1, \ldots, n$, then the sufficient condition of Corollary 4 for existence of a global attractor $x^{*}>0$ is that $\rho(A)<1$.

\section{CONCLUSION}

A feature often used in the stability analysis of nonlinear (interconnected) systems is that the nonlinearities are monotone and "declining", meaning, depending on the context, bounded or unbounded sigmoidals, or saturated and without inflection points, or scalable. For positive systems, a natural characterization of this feature is in terms of monotone and concave vector fields. For them, existence, uniqueness and stability of the (nontrivial) equilibrium point can be investigated efficiently, and reformulated as spectral conditions on the Jacobian of the system. The same spectral conditions allow to impose a positive attractor through simple stabilizing feedback laws.

A possible extension of our work deals with studying the behavior of nonlinear concave/subhomogeneous systems on the entire $\mathbb{R}^{n}$, rather than just on the positive orthant. From the results of [2], [3], [12], is clear that in this case the phase plane of the positive orthant is replicated in the negative orthant $\left(\mathbb{R}_{-}^{n}\right)$. It remains however to understand to what extent the spectral conditions developed here can be extended beyond the positive/negative orthant.
Acknoledgments. The authors would like to thank the reviewers for pointing out relevant references such as [23], [25], [32], [30].

\section{APPENDIX}

\section{A. Proof of Theorem 3}

From conditions 2, 4 and 5 of the theorem, we can apply Theorem 2, thus obtaining the existence of a fixed point. To show uniqueness of the fixed point, suppose $x>0$ is any fixed point of $f$. Consider $y>0$ such that $g(y)=f(y)-y \geqslant 0$. Let

$$
\alpha=\min \left\{\frac{x_{j}}{y_{j}}, j=1, \ldots, n\right\}=\frac{x_{r}}{y_{r}} .
$$

Then $\alpha>0$ because $x>0$ and $y>0$. If $\alpha \geqslant 1$ then it must be $y_{j} \leqslant x_{j} \forall j=1, \ldots, n$, hence $y \leqslant x$. Otherwise let $w=\alpha y$. Since $g$ is strictly subhomogeneous and $g(y) \geqslant 0$, we have that $g(\alpha y)>\alpha^{\tau} g(y)$ for $0<\alpha<1$, which implies $g(w)>0$. Then $w \leqslant x$ and $w_{r}=x_{r}$, so $g_{r}(x)-g_{r}(w)=$ $f_{r}(x)-f_{r}(w) \geqslant 0$ because $f$ is increasing. But this implies $0=g_{r}(x) \geqslant g_{r}(w)>0$, a contradiction. Thus $y>0$ and $g(y) \geqslant 0$ implies $y \leqslant x$. Now if $y>0$ is a fixed point of $f$ then, since $g(x)=0$, the same argument with the roles of $x$ and $y$ reversed gives $x \leqslant y$, so $y=x$.

\section{B. Proof of Theorem 4 and corollaries}

In order to prove Theorem 4 we will need the following lemma:

Lemma 5 Let $f: \mathbb{R}_{+}^{n} \rightarrow \mathbb{R}_{+}^{n}$ be a $C^{1}$, strictly concave and increasing vector field, $f(0)=0$. Assume $F(x) \geqslant 0$ and irreducible $\forall x \in \mathbb{R}_{+}^{n}$. Then

1) $\rho(F(0))>1$ if and only if $\exists x_{1} \in \operatorname{int}\left(\mathbb{R}_{+}^{n}\right)$ such that $f\left(x_{1}\right)>x_{1}$.

2) $\exists \bar{x} \in \operatorname{int}\left(\mathbb{R}_{+}^{n}\right)$ such that $\rho(F(\bar{x}))<1$ if and only if $\exists x_{2} \in \operatorname{int}\left(\mathbb{R}_{+}^{n}\right)$, such that $f\left(x_{2}\right)<x_{2}$.

Proof.

[Proof of case 1)] By contradiction, suppose that $\rho(F(0))=$ $\rho_{0} \leqslant 1$ and that $\exists x_{1} \in \mathbb{R}_{+}^{n}, x_{1}>0$ such that $f\left(x_{1}\right)>x_{1}$. Let $w_{0}$ be the left eigenvector of $F(0)$ corresponding to $\rho_{0}$. Since $F(0) \geqslant 0$ and irreducible, from the Perron-Frobenius theorem, $w_{0}>0$. Let $g: \mathbb{R}_{+}^{n} \rightarrow \mathbb{R}^{n}$ be defined as $g(x)=f(x)-x$, of Jacobian $G(x)=-I+F(x)$. From the strict concavity of $f$, also $g$ is strictly concave since it is a linear combination of concave functions. Since $f(x)>x \Longleftrightarrow g(x)>0$, from (4) the following relationship holds

$$
g\left(x_{1}\right)<g(0)+G(0) x_{1}=(-I+F(0)) x_{1} .
$$

Multiplying both sides by $w_{0}^{T}$ the inequality becomes

$$
w_{0}^{T} g\left(x_{1}\right)<-w_{0}^{T} x_{1}+w_{0}^{T} F(0) x_{1}=-w_{0}^{T} x_{1}+\rho_{0} w_{0}^{T} x_{1},
$$

or

$$
w_{0}^{T}\left(g\left(x_{1}\right)+x_{1}-\rho_{0} x_{1}\right)<0 .
$$

Since $w_{0}>0$, and defining $\epsilon$ as $\epsilon:=1-\rho_{0} \geqslant 0$, (24) implies that $\exists i \in\{1, \ldots, n\}$ such that

$$
g_{i}\left(x_{1}\right)+\left(1-\rho_{0}\right) x_{1, i}<0 \Longrightarrow g_{i}\left(x_{1}\right)<-\epsilon x_{1, i} \leqslant 0,
$$


which is a contradiction, since our hypothesis implies $x_{1, i}>0$ and $g_{i}\left(x_{1}\right)>0$ for all $i$. Now suppose $\rho(F(0))=\rho_{0}>1$. We want to show that there exists $x_{1}>0$ such that $f\left(x_{1}\right)>x_{1}$. From Taylor's theorem and the $C^{1}$ assumption for $f$, we have that for every $i=1, \ldots, n$ the following holds $\forall x_{0} \in \mathbb{R}_{+}^{n}$

$$
f_{i}(x)=f_{i}\left(x_{0}\right)+\frac{\partial f_{i}\left(x_{0}\right)}{\partial x}\left(x-x_{0}\right)+\eta_{i}\left(x-x_{0}\right),
$$

where $\eta_{i}: \mathbb{R}^{n} \rightarrow \mathbb{R}$ is such that

$$
\lim _{x \rightarrow x_{0}} \frac{\eta_{i}\left(x-x_{0}\right)}{\left\|x-x_{0}\right\|}=0 .
$$

Joining all $i=1, \ldots, n$ equations, yields

$$
f(x)=f\left(x_{0}\right)+F\left(x_{0}\right)\left(x-x_{0}\right)+\eta\left(x-x_{0}\right) .
$$

Let $v_{0}>0$ be the right eigenvector corresponding to the Perron-Frobenius eigenvalue $\rho(F(0))=\rho_{0}>1$. From Taylor's approximation in (25), by choosing $x_{0} \equiv 0$ and $x \equiv x_{1}$, we have

$$
f\left(x_{1}\right)=f(0)+F(0) x_{1}+\eta\left(x_{1}\right) .
$$

Since we are interested in finding a vector $x_{1}>0$ such that $f\left(x_{1}\right)>x_{1}$, let us choose $x_{1}=\gamma v_{0}, \gamma>0$. The vector $x_{1}$ is clearly a positive vector and

$$
\lim _{\gamma \rightarrow 0} \frac{\eta\left(x_{1}\right)}{\gamma}=0 .
$$

With these choices, equation (26) becomes

$$
f\left(x_{1}\right)=\rho(F(0)) x_{1}+\eta\left(x_{1}\right)=\rho_{0} x_{1}+\eta\left(x_{1}\right)
$$

or, rewriting $\rho_{0}$ as $\rho_{0}=1+\epsilon, \epsilon>0$,

$$
f\left(x_{1}\right)=x_{1}+\epsilon x_{1}+\eta\left(x_{1}\right) .
$$

Recalling that $x_{1}=\gamma v_{0}$, it is

$$
f\left(x_{1}\right)=x_{1}+\gamma\left(\epsilon v_{0}+\frac{1}{\gamma} \eta\left(x_{1}\right)\right)
$$

and, from (27), $\epsilon v_{0}+(1 / \hat{\gamma}) \eta\left(x_{1}\right)>0$ for an appropriate small $\hat{\gamma} \neq 0$, or

$$
f\left(x_{1}\right)=x_{1}+\hat{\gamma}(\text { something positive })>x_{1},
$$

which completes the proof of this first part.

[Proof of case 2)]. Suppose there $\exists \bar{x} \in \mathbb{R}_{+}^{n}$ such that $\rho(F(\bar{x}))=\bar{\rho}<1$. We first show that there exists $x_{2} \in \mathbb{R}_{+}^{n}$, such that $f\left(x_{2}\right)<x_{2}$. Consider again $g(x)=f(x)-x$. We assume $\exists i \in\{1, \ldots, n\}$ such that $g_{i}(\bar{x}) \geqslant 0$, otherwise the proof would be finished since $g(\bar{x})<0$ implies $f(\bar{x})<\bar{x}$. Since $F(\bar{x}) \geqslant 0$ and irreducible, from the Perron-Frobenius theorem we have that $\bar{\rho}$ is a real positive eigenvalue of $F(\bar{x})$ and its right eigenvector, call it $v$, must be positive, $v>0$. Let us define $\epsilon$ as $\epsilon=1-\bar{\rho}$, clearly $\epsilon>0$ since $\bar{\rho}<1$. Furthermore, since the Jacobian matrix of $g$ in $\bar{x}$ is $G(\bar{x})=-I+F(\bar{x})$, it is easily seen that $v$ is also the right eigenvector of $G(\bar{x})$ relative to $-\epsilon$ :

$$
G(\bar{x}) v=(-I+F(\bar{x})) v=(-1+\bar{\rho}) v=-\epsilon v .
$$

We need to show that there exists $x_{2}$ such that $g\left(x_{2}\right)<0$. Let us define $x_{2}$ as

$$
x_{2}=\bar{x}+\gamma v \quad \gamma \in \mathbb{R}_{+}, \gamma>0 .
$$

As $v>0$ and $\gamma$ is positive, it is clear that $x_{2}>\bar{x}$. The vector field $g$ is strictly concave. Then, from (4), one gets

$$
g\left(x_{2}\right)<g(\bar{x})+G(\bar{x})\left(x_{2}-\bar{x}\right) .
$$

From (29) and (28), the previous expression becomes

$$
g\left(x_{2}\right)<g(\bar{x})-\epsilon \gamma v .
$$

By choosing an appropriate $\gamma$ the right hand side can be made negative. For example

$$
\gamma:=\frac{1}{\epsilon} \frac{\max _{i=1, \ldots, n}\left\{g_{i}(\bar{x})\right\}}{\min _{j=1, \ldots, n}\left\{v_{j}\right\}}
$$

implies $\left(\mathbf{1}=\left[\begin{array}{lll}1 & \ldots & 1\end{array}\right]^{T}\right)$

$$
\begin{aligned}
g\left(x_{2}\right) & <g(\bar{x})-\frac{\max _{i=1, \ldots, n}\left\{g_{i}(\bar{x})\right\}}{\min _{j=1, \ldots, n}\left\{v_{j}\right\}} v \\
& \leqslant g(\bar{x})-\max _{i=1, \ldots, n}\left\{g_{i}(\bar{x})\right\} \mathbf{1} \leqslant 0
\end{aligned}
$$

since $\left(v_{i} / \min _{j=1, \ldots, n}\left\{v_{j}\right\}\right) \geqslant 1, \forall i$. The proof of sufficiency is completed since $g\left(x_{2}\right)<0 \Longrightarrow f\left(x_{2}\right)<x_{2}$. To show the necessity part, assume $\exists x_{2} \in \mathbb{R}_{+}^{n}$, such that $f\left(x_{2}\right)<x_{2}$. To prove this part it is enough to choose $\bar{x}=x_{2}$. Assume by contradiction that $\rho\left(F\left(x_{2}\right)\right) \geqslant 1$. Then by strict concavity of $f$ the following holds

$$
f(0)<f\left(x_{2}\right)+F\left(x_{2}\right)\left(0-x_{2}\right)
$$

which, from $f(0)=0$ and $f\left(x_{2}\right)<x_{2}$, yields

$$
0<x_{2}-F\left(x_{2}\right) x_{2} .
$$

Let $w>0$ be the left Perron-Frobenius eigenvector corresponding to $\rho\left(F\left(x_{2}\right)\right)$. Multiplying both sides of (30) and rearranging

$$
w^{T} F\left(x_{2}\right) x_{2}=\rho\left(F\left(x_{2}\right)\right) w^{T} x_{2}<w^{T} x_{2}
$$

which is clearly a contradiction if $\rho\left(F\left(x_{2}\right)\right) \geqslant 1$.

Proof of Theorem 4. By using Lemma 5, the two conditions of Theorem 4 become:

1) $\exists x_{1} \in \operatorname{int}\left(\mathbb{R}_{+}^{n}\right)$ such that $f\left(x_{1}\right)>x_{1}$;

2) $\exists x_{2} \in \operatorname{int}\left(\mathbb{R}_{+}^{n}\right)$ such that $f\left(x_{2}\right)<x_{2}$.

When condition 1 holds, $x_{2}$ can always be chosen such that $x_{2}>x_{1}$ (since $x_{1} \in \operatorname{int}\left(\mathbb{R}_{+}^{n}\right)$ can be chosen arbitrary close to 0). Hence Theorem 3 applies and the system (7) admits a unique positive equilibrium.

Proof of Proposition 2. The proof is identical to the necessity part of the second condition of Lemma 5, provided one replaces " $f\left(x_{2}\right)<x_{2}$ " with " $f\left(x^{*}\right)=x^{*}$ ".

Proof of Proposition 3. Letting $g(x)=f(x)-x$, we need to show that if $g\left(x_{2}\right)<0$ then $g(x)<0 \forall x>x_{2}$. By contradiction, let us suppose that there exists $i \in\{1, \ldots, n\}$ such that

$$
g_{i}(x) \geqslant 0 \quad \text { for } \quad x>x_{2}
$$


Since $g_{i}$ is strictly concave, the upper contour set $S_{\alpha}=\left\{x \in \mathbb{R}_{+}^{n}: g_{i}(x) \geqslant \alpha\right\}$ must be convex for all $\alpha \in \mathbb{R}$. Choosing for example $\alpha=g_{i}\left(x_{2}\right) / 2$, it is clear that $x_{2} \notin S_{\alpha}$ while $x \in S_{\alpha}$. Let us define $\bar{z}$ as $\bar{z}=\bar{\lambda} x+(1-\bar{\lambda}) x_{2}$, where $\bar{\lambda}$ is the smallest real number in $(0,1)$ such that $\bar{z} \in S_{\alpha}$. Clearly $\alpha<0$, thus $0 \in S_{\alpha}$. Then, from the strict concavity of $g_{i}$, the convex combination of 0 and $\bar{z}$ should lie in $S_{\alpha}$, but $g(\beta \bar{z})<\alpha$ for some $\beta<1$. This shows that $S_{\alpha}$ is not convex. Thus it must be $g_{i}(x)<0$ i.e. $f(x)<x \forall x>x_{2}$.

Proof of Proposition 4. From strict concavity of $f$, the following function in $\xi$ is non-increasing

$$
\kappa(\xi)=\mathbf{1}^{T} F(x+\xi v) v
$$

where $\xi>0$ is a scalar and $v>0$ is a direction. For $\kappa(\xi)$ in fact

$$
\frac{\partial \kappa}{\partial \xi}(\xi)=\mathbf{1}^{T}\left(\begin{array}{c}
v^{T} \nabla^{2} f_{1}(x+\xi v) v \\
\vdots \\
v^{T} \nabla^{2} f_{n}(x+\xi v) v
\end{array}\right) \leqslant 0
$$

which follows from $v^{T} \nabla^{2} f_{i}(x+\xi v) v \leqslant 0 \forall \xi \geqslant 0$, since strict concavity of $f_{i}$ implies negative semidefiniteness of the Hessian matrix $\nabla^{2} f_{i}(x+\xi v) \forall \xi \geqslant 0$. However we would like to show that $\kappa$ is actually decreasing. To prove it, let $x_{1}=x+\xi_{1} v$ and $x_{2}=x_{1}+\xi_{2} v$ with $\xi_{2}>0$ and $\xi_{1}>0$. For strictly concave $f$, (4) implies $\forall x, y \in \mathbb{R}_{+}^{n}, x_{i} \neq y_{i}$

$$
F(x)(y-x)>F(y)(y-x) .
$$

Thus choosing $y=x+\xi v$ the previous equation is

$$
F(x) v>F(y) v .
$$

From these facts it is clear that $F(x) v>F\left(x_{1}\right) v>F\left(x_{2}\right) v$, thus our choices for $x_{1}$ and $x_{2}$ imply that $\kappa$ is decreasing. Let $w_{\xi}>0$ be the left Perron-Frobenius eigenvector corresponding to $\rho(F(x+\xi v))$. Without loss of generality, we can assume that $w_{\xi}^{T} \leqslant \mathbf{1}^{T}$ and that $\exists j \in\{1, \ldots, n\}$ such that $w_{\xi, j}=1$. With this assumptions

$$
\kappa(\xi) \geqslant w_{\xi}^{T} F(x+\xi v) v=\rho(F(x+\xi v)) w_{\xi}^{T} v
$$

and $w_{\xi}^{T} v>v_{j} \geqslant v_{\min }=\min _{i}\left\{v_{i}, i=1, \ldots, n\right\}$. This yields

$$
\kappa(\xi)>\rho(F(x+\xi v)) v_{\min } .
$$

The proof is concluded if we show that having an $f$ that is bounded, strict concave and monotone in turn implies

$$
\kappa(\xi)=\mathbf{1}^{T} F(x+\xi v) v \rightarrow 0, \xi \rightarrow \infty \forall v \geqslant 0, v \neq 0 .
$$

By contradiction, let us suppose there $\exists v$ such that $\kappa(\xi)=\mathbf{1}^{T} F(x+\xi v) v \geqslant \epsilon>0$ for $\xi \rightarrow \infty$. From strict concavity and (4), choosing $x_{1}=x$ and $x_{2}=x+\xi v$ we have

$$
f(x)<f(x+\xi v)-F(x+\xi v) \xi v .
$$

Thus, multiplying both sides by $\mathbf{1}^{T}$,

$$
\mathbf{1}^{T} f(x+\xi v)>\mathbf{1}^{T} f(x)+\xi \kappa(\xi)
$$

and for $\xi \rightarrow \infty, \mathbf{1}^{T} f(x+\xi v)$ can be made arbitrarily large. This is clearly a contradiction since $f$ is bounded. We have shown that $\kappa(\xi) \rightarrow 0$ for $\xi \rightarrow \infty$, thus from (31) and since $x, v$ are arbitrarily chosen we have

$$
\lim _{\|x\| \rightarrow \infty} \rho(F(x))=0 .
$$

\section{Proof of Theorem 5}

[Proof of case 1)] From the Perron-Frobenius theorem and from the irreducibility of $F(0), w_{0}$, the left eigenvector of $F(0)$ relative to $\rho_{0}=\rho(F(0))$ is $w_{0}>0$. For the system (7), the diagonal matrix $\Delta$ is positive definite which implies that $\Delta^{-1}$ is positive definite. Let $V: \mathbb{R}_{+}^{n} \rightarrow \mathbb{R}_{+}$be the following Lyapunov function

$$
V(x)=\frac{1}{2} x^{T}\left(\Delta^{-1} w_{0} w_{0}^{T} \Delta^{-1}\right) x .
$$

The matrix $\left(\Delta^{-1} w_{0} w_{0}^{T} \Delta^{-1}\right)$ is clearly symmetric and strictly positive, hence $V(x)>0 \forall x \neq 0, V(0)=0$. Differentiating $V$ we have

$$
\begin{aligned}
\dot{V}(x) & =x^{T} \Delta^{-1} w_{0} w_{0}^{T} \Delta^{-1} \dot{x} \\
& =x^{T} \Delta^{-1} w_{0} w_{0}^{T}(-x+f(x)) .
\end{aligned}
$$

From strict concavity of $f$ and from (4) (with $x_{1}=x$ and $x_{2}=0$ ), we have

$$
f(x) \leqslant F(0) x
$$

and there $\exists i$ such that $f_{i}(x)<[F(0) x]_{i}$. Therefore, multiplying both sides of the previous inequality by $w_{0}^{T}$ we obtain

$$
w_{0}^{T} f(x)<\rho(F(0)) w_{0}^{T} x=\rho_{0} w_{0}^{T} x .
$$

The assumption $\rho_{0}<1$ gives

$$
w_{0}^{T} f(x)<w_{0}^{T} x
$$

hence in (33) we have

$$
\begin{aligned}
\dot{V} & =-x^{T} \Delta^{-1} w_{0} w_{0}^{T} x+x^{T} \Delta^{-1} w_{0} w_{0}^{T} f(x) \\
& =x^{T} \Delta^{-1} w_{0}\left(-w_{0}^{T} x+w_{0}^{T} f(x)\right) .
\end{aligned}
$$

From condition (34), $-w_{0}^{T} x+w_{0}^{T} f(x)<0$, which implies that $\dot{V}<0$ for all $x \in \mathbb{R}_{+}^{n} \backslash\{0\}$, since $x^{T} \Delta^{-1} w_{0}>0$. The proof holds globally in $\mathbb{R}_{+}^{n}$ since $V$ is radially unbounded.

[Proof of case 2)] Under the hypothesis of case 2, existence and uniqueness of the equilibrium $x^{*} \in \operatorname{int}\left(\mathbb{R}_{+}^{n}\right)$ follow from Theorem 4. Given $x^{*}$, split $\mathbb{R}_{+}^{n}$ into the regions

$$
\begin{aligned}
& \Omega_{1}=\left\{x \in \mathbb{R}_{+}^{n} \text { such that } x \leqslant x^{*}\right\} \\
& \Omega_{2}=\left\{x \in \mathbb{R}_{+}^{n} \text { such that } x \geqslant x^{*}\right\} \\
& \Omega_{3}=\mathbb{R}_{+}^{n} \backslash\left(\Omega_{1} \cup \Omega_{2}\right) .
\end{aligned}
$$

From cooperativity (and Lemma 4 ) we have that $\Omega_{1}$ and $\Omega_{2}$ are forward invariant. In fact, the monotonicity property

$$
x_{0} \leqslant y_{0} \Longrightarrow x\left(t, x_{0}\right) \leqslant x\left(t, y_{0}\right) \quad \forall t \geqslant 0
$$

implies that on $\Omega_{1}$ we have

$$
x_{0} \leqslant x^{*} \Longrightarrow x\left(t, x_{0}\right) \leqslant x\left(t, x^{*}\right)=x^{*} \quad \forall t \geqslant 0
$$

and on $\Omega_{2}$

$$
x_{0} \geqslant x^{*} \Longrightarrow x\left(t, x_{0}\right) \geqslant x\left(t, x^{*}\right)=x^{*} \quad \forall t \geqslant 0 .
$$


If $g=-x+f(x)$, from Lemma 5, the assumption $\rho(F(0))>$ 1 implies that we can choose $a$ such that $f(a)>a$, and $a$ in an arbitrarily small neighbourhood of the origin, i.e., $a \in \Omega_{1}$ and $g(a)>0$. From the assumption $\rho\left(F\left(x_{2}\right)\right)<1$ we can choose $b$ such that $f(b)<b$, with $b$ arbitrarily large and such that $b \in \Omega_{2}$ and $g(b)<0$. Consider the Lyapunov function

$$
V(x)=\frac{1}{2}\left(x-x^{*}\right)^{T} \Delta^{-1}\left(x-x^{*}\right) .
$$

Clearly $V(x)>0 \forall x \in \mathbb{R}_{+}^{n} \backslash\left\{x^{*}\right\}, V\left(x^{*}\right)=0$. From Lemma 4 and $g(a)>0$ we have that

$$
\dot{V}(x(t, a))=\left(x(t, a)-x^{*}\right)^{T} \Delta^{-1} \dot{x}(t, a)<0 \forall t \geqslant 0
$$

since $a \in \Omega_{1} \Rightarrow x(t, a) \in \Omega_{1}$ and $\dot{x}(t, a)>0 \forall t \geqslant 0$, i.e, $x(t, a) \rightarrow x^{*}$. Analogously from $g(b)<0, \dot{V}(x(t, b))<$ $0 \forall t \geqslant 0$, thus $x(t, b) \rightarrow x^{*}$. Now $\forall x_{0} \in \operatorname{int}\left(\mathbb{R}_{+}^{n}\right)$ the vectors $a$ and $b$ can be chosen so that $a \leqslant x_{0} \leqslant b$. It then follows from the monotonicity property that $\forall t \geqslant 0$

$$
a \leqslant x_{0} \leqslant b \Longrightarrow x(t, a) \leqslant x\left(t, x_{0}\right) \leqslant x(t, b) .
$$

Since we already know that $x(t, a) \rightarrow x^{*}$ and that $x(t, b) \rightarrow$ $x^{*}$, it must be $x\left(t, x_{0}\right) \rightarrow x^{*}$, i.e., $x^{*}$ is asymptotically stable. If $x_{0} \in \mathrm{bd}\left(\mathbb{R}_{+}^{n}\right)$ then irreducibility and cooperativity imply that $x\left(t, x_{0}\right) \in \operatorname{int}\left(\mathbb{R}_{+}^{n}\right)$ for $t>0$, thus convergence to $x^{*}$. Since $V(x)$ is radially unbounded, convergence to $x^{*}$ is global in $\mathbb{R}_{+}^{n} \backslash\{0\}$.

\section{Proof of Theorem 6}

The following lemma is instrumental to the proof of Theorem 6 .

Lemma 6 Let $f: \mathbb{R}_{+}^{n} \rightarrow \mathbb{R}_{+}^{n}$ a $C^{1}$ vector field which is strictly subhomogeneous of degree $0<\tau \leqslant 1$ and increasing. Assume $f(0)=0$ and $F(x) \geqslant 0$ irreducible $\forall x \in \mathbb{R}_{+}^{n}$.

1) If $\rho(F(0))>1$ then $\exists x_{1} \in \operatorname{int}\left(\mathbb{R}_{+}^{n}\right)$ such that $f\left(x_{1}\right)>$ $x_{1}$.

2) If $\exists \bar{x} \in \operatorname{int}\left(\mathbb{R}_{+}^{n}\right)$ such that $\rho(F(x)) \leqslant \zeta_{0}<1 \forall x \geqslant \bar{x}$, then $\exists x_{2} \in \operatorname{int}\left(\mathbb{R}_{+}^{n}\right)$ such that $f\left(x_{2}\right)<x_{2}$.

\section{Proof.}

[Proof of case 1)] It is omitted because completely analogous to part 1 of Lemma 5.

[Proof of case 2)] Suppose there exists $\bar{x} \in \mathbb{R}_{+}^{n}$ such that $\rho(F(\bar{x})) \leqslant \zeta_{0}<1$ and let $g$ be $g(x)=f(x)-x$. Denote $\tilde{x}_{0}=\bar{x}$. From Taylor's theorem and from the differentiability assumptions for $f, g$ is differentiable and the following holds

$$
g(x)=g\left(\tilde{x}_{0}\right)+G\left(\tilde{x}_{0}\right)\left(x-\tilde{x}_{0}\right)+\eta\left(x-\tilde{x}_{0}\right) .
$$

where each component $\eta_{j}: \mathbb{R}^{n} \rightarrow \mathbb{R}$ of $\eta$ is such that

$$
\lim _{x \rightarrow \tilde{x}_{0}} \frac{\eta_{j}\left(x-\tilde{x}_{0}\right)}{\left\|x-\tilde{x}_{0}\right\|}=0 .
$$

Let us define $\tilde{x}_{1} \in \mathbb{R}_{+}^{n}, \tilde{x}_{1}>\tilde{x}_{0}$ as

$$
\tilde{x}_{1}=\tilde{x}_{0}+\gamma_{0} v_{0}, \quad \gamma_{0}>0
$$

where $v_{0}>0,\left\|v_{0}\right\|=1$, is the positive PerronFrobenius eigenvalue of $F\left(\tilde{x}_{0}\right)$ corresponding to the eigenvalue $\rho\left(F\left(\tilde{x}_{0}\right)\right)$. Equivalently

$$
G\left(\tilde{x}_{0}\right) v_{0}=\left(-1+\rho\left(F\left(\tilde{x}_{0}\right)\right)\right) v_{0}=-\epsilon_{0} v_{0}
$$

where $\epsilon_{0}=1-\rho\left(F\left(\tilde{x}_{0}\right)\right)$ is a positive constant since $\rho\left(F\left(\tilde{x}_{0}\right)\right) \leqslant \zeta_{0}<1$. From Taylor's approximation, the following holds

$$
g\left(\tilde{x}_{1}\right)-g\left(\tilde{x}_{0}\right)=-\epsilon_{0} \gamma_{0} v_{0}+\eta\left(\tilde{x}_{1}-\tilde{x}_{0}\right)
$$

and, since $\frac{1}{\gamma_{0}} \eta\left(\tilde{x}_{1}-\tilde{x}_{0}\right) \rightarrow 0$ for $\gamma_{0} \rightarrow 0$, it is always possible to chose $\gamma_{0} \equiv \hat{\gamma}_{0}>0$ appropriately small so that in correspondence $-\epsilon_{0} \gamma_{0} v_{0}+\eta\left(\tilde{x}_{1}-\tilde{x}_{0}\right) \leqslant \varpi<0$ for a given $\varpi$. This means that $g\left(\tilde{x}_{1}\right)<g\left(\tilde{x}_{0}\right)$. If $g\left(\tilde{x}_{1}\right)=$ $g\left(\tilde{x}_{0}\right)-\epsilon_{0} \gamma_{0} v_{0}+\eta\left(\tilde{x}_{1}-\tilde{x}_{0}\right)<0$ the proof is concluded once we choose $x_{2} \equiv \tilde{x}_{1}$. Otherwise we iterate the procedure defining $\tilde{x}_{i+1}>\tilde{x}_{i}$ as

$$
\tilde{x}_{i+1}=\tilde{x}_{i}+\gamma_{i} v_{i}, \quad \gamma_{i}>0
$$

where $v_{i}>0,\left\|v_{i}\right\|=1$, is the positive Perron-Frobenius eigenvalue of $F\left(\tilde{x}_{i}\right)$, with $\rho\left(F\left(\tilde{x}_{i}\right)\right) \leqslant \zeta_{0}<1$ by assumption. In this way we obtain an increasing sequence $\tilde{x}_{0}<\tilde{x}_{1}<$ $\ldots<\tilde{x}_{i}<\tilde{x}_{i+1}$, which, for suitably small $\hat{\gamma}_{i}>0$, obeys to an expression similar to (37)

$$
g\left(\tilde{x}_{i+1}\right)-g\left(\tilde{x}_{i}\right)=-\epsilon_{i} \hat{\gamma}_{i} v_{i}+\eta_{i}\left(\tilde{x}_{i+1}-\tilde{x}_{i}\right) \leqslant \varpi<0 .
$$

Summing terms up to the $N$-th iteration, the following holds by construction $\left(\eta_{0}=\eta\right)$

$g\left(\tilde{x}_{N}\right)-g\left(\tilde{x}_{0}\right)=\sum_{i=0}^{N-1}\left(-\epsilon_{i} \hat{\gamma}_{i} v_{i}+\eta_{i}\left(\tilde{x}_{i+1}-\tilde{x}_{i}\right)\right) \leqslant N \varpi<0$.

From the assumption that $\rho(F(x)) \leqslant \zeta_{0}<1 \forall x>\bar{x}$, it follows that for all terms $\tilde{x}_{0}, \ldots, \tilde{x}_{i}, \ldots, \tilde{x}_{N}$ it is $\hat{\gamma}_{i} \geqslant \hat{\gamma}_{\min }>0$, where $\hat{\gamma}_{\text {min }}$ is small but finite, and in correspondence (38) holds for all $i$. Hence for any $g\left(\tilde{x}_{0}\right)$, from (39), there exists $N$ large enough for which $N \varpi<-g\left(\tilde{x}_{0}\right)$, and therefore $g\left(\tilde{x}_{N}\right)<0$. The Lemma holds once we choose $x_{2}=\tilde{x}_{N}$.

Proof of Theorem 6. Using Lemma 6, the two conditions of Theorem 6 become

1) $\exists x_{1} \in \operatorname{int}\left(\mathbb{R}_{+}^{n}\right)$ such that $f\left(x_{1}\right)>x_{1}$;

2) $\exists x_{2} \in \operatorname{int}\left(\mathbb{R}_{+}^{n}\right)$ such that $f\left(x_{2}\right)<x_{2}$.

Provided we choose $x_{1}<x_{2}$ (always possible as $x_{1}$ can be arbitrarily close to 0 ), then Theorem 3 applies and the existence and uniqueness follow. To shown stability, from strict subhomogeneity of $f$ of degree $0<\tau \leqslant 1$ and invoking Lemma 3, we have that $f$ is also subhomogeneous of degree 1 , hence

$$
\begin{aligned}
& f(\lambda x)<\lambda^{\tau} f(x) \leqslant \lambda f(x), \quad \forall \lambda>1 \\
& f(\alpha x)>\alpha^{\tau} f(x) \geqslant \alpha f(x), \quad 0<\alpha<1 .
\end{aligned}
$$

Subtracting $-\lambda x$ from both sides of the first of (40) and $-\alpha x$ from both sides of the second we obtain

$$
\begin{array}{ll}
-\lambda x+f(\lambda x)<-\lambda x+\lambda f(x), & \forall \lambda>1 \\
-\alpha x+f(\alpha x)>-\alpha x+\alpha f(x), & 0<\alpha<1,
\end{array}
$$


which are equivalent to

$$
\begin{aligned}
& g(\lambda x)<\lambda g(x), \quad \forall \lambda>1, \\
& g(\alpha x)>\alpha g(x), \quad 0<\alpha<1 .
\end{aligned}
$$

From $g\left(x^{*}\right)=0$ and $\rho\left(F\left(x^{*}\right)\right)<1$ (see proof of uniqueness above), $\mu\left(G\left(x^{*}\right)\right)<0$, i.e., $G\left(x^{*}\right)$ is Hurwitz in $x^{*}$ and we can find $b>x^{*}$ such that $g(b)<0$ and $a<x^{*}$ such that $g(a)>0$. From Taylor's approximation it can be for example $b=x^{*}+\gamma v_{0}, a=x^{*}-\gamma v_{0}$ for an appropriate small $\gamma$. Strict subhomogeneity, $g(b)<0$ and $g(a)>0$ imply then

$$
\begin{aligned}
& g(\lambda b)<\lambda g(b)<0, \quad \forall \lambda>1 \\
& g(\alpha a)>\alpha g(a)>0, \quad \forall \alpha \in(0,1) .
\end{aligned}
$$

Now, if $G(x)$ is cooperative and irreducible $\forall x>0$, Lemma 4 applies and, analogously to the proof of Theorem 5 (condition 2), the two regions $\Omega_{1}$ and $\Omega_{2}$ in (35) are forward invariant for (7). Let us consider the Lyapunov function (36). From (41), choosing $\bar{\lambda}>1, x(t, \bar{\lambda} b) \in \Omega_{2} \forall t \geqslant 0$, i.e., $x(t, \bar{\lambda} b)>x^{*}$ $\forall t \geqslant 0$, hence $g(\bar{\lambda} b)<0$ and $\dot{x}(t, \bar{\lambda} b)<0$. Differentiating $V$ along this trajectory, we have therefore

$$
\dot{V}(x(t, \bar{\lambda} b))=\left(x(t, \bar{\lambda} b)-x^{*}\right)^{T} \Delta^{-1} \dot{x}(t, \bar{\lambda} b)<0 .
$$

The case $x \in \Omega_{1}$ is analogous, and the rest of the proof follows along similar lines of the proof of condition 2 of Theorem 5 .

\section{E. Proof of Proposition 5}

From the monotonicity condition of c-contractive interference functions, we have that the following holds

$$
\Phi(x)=\left(\begin{array}{c}
\nabla \phi_{1}(x) \\
\vdots \\
\nabla \phi_{n}(x)
\end{array}\right) \geqslant 0 .
$$

Furthermore, in the contractivity condition (8), $\epsilon>0$ can be chosen arbitrarily. In particular we can make the choice $\epsilon=$ $\gamma /\|\nu\|, \gamma>0$. The inequality in (8), which holds for every component of $\phi$ and for every $\gamma>0$, i.e. $\forall i=1, \ldots, n$,

$$
\phi_{i}(x+\epsilon \nu) \leqslant \phi_{i}(x)+c \epsilon \nu_{i},
$$

with our choice of $\epsilon$ becomes

$$
\phi_{i}\left(x+\gamma \frac{\nu}{\|\nu\|}\right) \leqslant \phi_{i}(x)+c \gamma \frac{\nu_{i}}{\|\nu\|} .
$$

By rearranging the inequality and defining $u=\nu /\|\nu\|$ as a unit vector (note that $u>0$ since $\nu>0$ by assumption), we have

$$
\frac{\phi_{i}(x+\gamma u)-\phi_{i}(x)}{\gamma} \leqslant c u_{i} .
$$

From the differentiability of $\phi_{i}$ in $x$ and taking the limit for $\gamma \rightarrow 0$ we obtain the following

$$
\lim _{\gamma \rightarrow 0} \frac{\phi_{i}(x+\gamma u)-\phi_{i}(x)}{\gamma}=\nabla \phi_{i}(x) \cdot u,
$$

which is the directional derivative along $u$. The contractivity condition is then

$$
\nabla \phi_{i}(x) \cdot u \leqslant c u_{i}
$$

The previous inequality holds for all $i=1, \ldots, n$, so it yields

$$
\Phi(x) u \leqslant c u .
$$

Since the Jacobian matrix is non-negative $\forall x \in \mathbb{R}_{+}^{n}$, it must be

$$
\rho(\Phi(x)) \geqslant 0
$$

and there exists a non-zero left eigenvector $w \in \mathbb{R}_{+}^{n}, w \neq 0$ such that

$$
w^{T} \Phi(x)=\rho(\Phi(x)) w^{T},
$$

so from this last equation, together with the inequality in (42), we have

$$
\rho(\Phi(x)) w^{T} u \leqslant c w^{T} u .
$$

Since $u>0$ and $w^{T} \geqslant 0, w \neq 0$, we have that $w^{T} u$ is a real positive value. Furthermore $c$ is a positive constant less that 1. The inequality is then

$$
\rho(\Phi(x))<1
$$

which concludes the proof.

\section{F. Proof of Proposition 6}

Consider Taylor's expansion

$$
\phi(x+\epsilon \nu)=\phi(x)+\Phi(x) \epsilon \nu+\eta(\epsilon \nu),
$$

where $\eta$ contains second and higher order terms in $\epsilon$, and let $\nu=\gamma v_{0}$, where $\gamma>0$ and $v_{0}$ is the right Perron-Frobenius eigenvector of $\Phi(x)$ corresponding to the eigenvalue $\rho(\Phi(x))$. The last equation becomes

$$
\phi(x+\epsilon \nu)=\phi(x)+\epsilon \rho(\Phi(x)) \gamma v_{0}+\eta\left(\epsilon \gamma v_{0}\right) .
$$

From Definition $1, \phi$ is c-contractive if for some $c \in[0,1)(8)$ holds. If we define $c$ as

$$
c=\zeta_{0}+\frac{1-\zeta_{0}}{2}
$$

for some $\zeta_{0}$ such that

$$
\rho(\Phi(x)) \leqslant \zeta_{0}<1
$$

from (43) we can guarantee the contractivity condition if

$$
\epsilon \rho(\Phi(x)) \gamma v_{0}+\eta\left(\epsilon \gamma v_{0}\right) \leqslant\left(\zeta_{0}+\frac{1-\zeta_{0}}{2}\right) \epsilon \gamma v_{0},
$$

Clearly $1>c>\zeta_{0}$. Inequality (46) is then

$$
\gamma\left((c-\rho(\Phi(x))) \epsilon v_{0}+\frac{\eta\left(\epsilon \gamma v_{0}\right)}{\gamma}\right) \geqslant 0 .
$$

By assumption for $\rho(\Phi(x))$ we have that $c-\rho(\Phi(x))>0$ is a positive scalar for all $x \in \mathbb{R}_{+}^{n}$. From Taylor's approximation $\eta \rightarrow 0$ if $\gamma \rightarrow 0$, and inequality (47) is satisfied if $v_{0}>0$, that is if $\Phi(x)$ is irreducible. In conclusion, under conditions (45) and $\Phi(x)$ irreducible for all $x \in \mathbb{R}_{+}^{n}$, $\phi$ is contractive, i.e. (8) holds with $\nu=\gamma v_{0}$, for an appropriate $\gamma>0$ and $c$ defined as is (44). 


\section{G. Proof of Theorem 7}

From Proposition 6, $f$ is a c-contractive interference function. Thus, Theorem 3 of [10] implies asymptotic stability of the unique positive equilibrium point.

\section{H. Proof of Theorem 8 and corollaries}

Proof of Theorem 8. First observe that, since $F(x) \geqslant 0$ and irreducible, it must be $\rho(\tilde{F}(x))>0$, hence both conditions of the Theorem require $k_{\min }>0$. We can therefore restrict to $K$ invertible. Then invertibility and nonnegativity of $K$ imply that the closed-loop system (9)-(10) can be rewritten as

$$
\dot{x}(t)=K\left(-x(t)+K^{-1} \tilde{f}(x(t))\right),
$$

which, by construction, is still a cooperative system, and hence is positive. Let us focus on the second statement of the Theorem. Defining $f$ as $f(x(t))=K^{-1} \tilde{f}(x(t))$, we can readily apply Theorems 4 and 5 to (48). It follows that the conditions

$$
\rho(F(0))>1
$$

and

$$
\rho\left(F\left(x_{2}\right)\right)<1 \text { for some } x_{2} \in \operatorname{int}\left(\mathbb{R}_{+}^{n}\right)
$$

hold for (48). In order to avoid the dependence from $K$ in $F$, we must obtain conditions like (49) and (50) directly in terms of $\tilde{F}$. Let $v_{0}>0$ be the Perron-Frobenius right eigenvector corresponding to the eigenvalue $\rho(F(0))$ and $\tilde{w}_{0}>0$ be the Perron-Frobenius left eigenvector corresponding to the eigenvalue $\rho(\tilde{F}(0))$, i.e.

$$
\begin{aligned}
& F(0) v_{0}=\rho(F(0)) v_{0}, \\
& \tilde{w}_{0}^{T} \tilde{F}(0)=\tilde{w}_{0}^{T} \rho(\tilde{F}(0)) .
\end{aligned}
$$

From the definition of $f$ the following equivalences holds:

$$
F(0)=K^{-1} \tilde{F}(0) \Rightarrow F(0) v_{0}=K^{-1} \tilde{F}(0) v_{0}
$$

or

$$
K \rho(F(0)) v_{0}=\tilde{F}(0) v_{0} .
$$

Multiplying both sides of (52) by $\tilde{w}_{0}^{T}$ we obtain

$$
\rho(F(0)) \tilde{w}_{0}^{T} K v_{0}=\tilde{w}_{0}^{T}, \tilde{F}(0) v_{0}
$$

which, from (51), yields

$$
\rho(F(0)) \tilde{w}_{0}^{T} K v_{0}=\rho(\tilde{F}(0)) \tilde{w}_{0}^{T} v_{0} .
$$

From $v_{0}>0, \tilde{w}_{0}^{T}>0$ we have $\tilde{w}_{0}^{T} v_{0}>0$ and $\tilde{w}_{0}^{T} K v_{0}>0$. Therefore

$$
\rho(F(0))=\rho(\tilde{F}(0)) \frac{\tilde{w}_{0}^{T} v_{0}}{\tilde{w}_{0}^{T} K v_{0}} .
$$

Condition (49) is then equivalent to

$$
\rho(\tilde{F}(0))>\frac{\tilde{w}_{0}^{T} K v_{0}}{\tilde{w}_{0}^{T} v_{0}} .
$$

Analogously, condition (50) is equivalent to

$$
\rho\left(\tilde{F}\left(x_{2}\right)\right)<\frac{\tilde{w}_{2}^{T} K v_{2}}{\tilde{w}_{2}^{T} v_{2}},
$$

where $\tilde{w}_{2}^{T}$ and $v_{2}$ are respectively the left and right PerronFrobenius eigenvectors corresponding to the eigenvalues $\rho\left(\tilde{F}\left(x_{2}\right)\right)$ and $\rho\left(F\left(x_{2}\right)\right)$. The new conditions in (54) and (55) are difficult to satisfy because the four eigenvectors depends on the Jacobian matrix of $f$ and $\tilde{f}$. To get rid of these intrinsic dependences, let us consider the term $\frac{\tilde{w}_{0}^{T} K v_{0}}{\tilde{w}_{0}^{T} v_{0}}$, which expanded yields

$$
\frac{\tilde{w}_{0}^{T} K v_{0}}{\tilde{w}_{0}^{T} v_{0}}=\frac{1}{\tilde{w}_{0}^{T} v_{0}} \sum_{i=1}^{n} k_{i} \tilde{w}_{0, i} v_{0, i} .
$$

Multiplying the right side by $k_{\max } / k_{\max }$ we obtain

$$
\frac{\tilde{w}_{0}^{T} K v_{0}}{\tilde{w}_{0}^{T} v_{0}}=\frac{k_{\max }}{\tilde{w}_{0}^{T} v_{0}} \sum_{i=1}^{n} \frac{k_{i}}{k_{\max }} \tilde{w}_{0, i} v_{0, i} \leqslant k_{\max }
$$

since $\frac{k_{i}}{k_{\max }} \leqslant 1$ for $i=1, \ldots, n$. Therefore the condition

$$
\rho(\tilde{F}(0))>k_{\max }
$$

is sufficient to guarantee (54) and hence (49). Analogously, the condition

$$
\rho\left(\tilde{F}\left(x_{2}\right)\right)<k_{\min }
$$

is sufficient to guarantee (55) and hence (50), which concludes the proof of the second condition of the Theorem. The proof of the first condition can be carried out in an analogous way.

Proof of Corollary 2. From (48), the condition $k_{\min }>$ $\rho(\tilde{F}(x))$ can be written as

$$
1>\frac{1}{k_{\min }} \rho(\tilde{F}(x)) \geqslant \rho\left(K^{-1} \tilde{F}(x)\right)
$$

meaning that $f(x)=K^{-1} \tilde{f}(x)$ is a c-contractive interference function. Theorem 7 then applies.

\section{Proof of Proposition 7}

$\psi_{j}$ strictly concave and non-decreasing implies that $\frac{\partial \psi_{j}\left(x_{j}\right)}{\partial x_{j}} \geqslant 0$ is decreasing (since $\frac{\partial^{2} \psi_{j}\left(x_{j}\right)}{\partial x_{j}^{2}}<0$ ). For $0<$ $x_{1}<x_{2}$ (of components $x_{1, j}$ and $x_{2, j}$ ) then

$$
\frac{\partial \psi_{j}\left(x_{1, j}\right)}{\partial x_{j}}>\frac{\partial \psi_{j}\left(x_{2, j}\right)}{\partial x_{j}} \geqslant 0 .
$$

Therefore $F\left(x_{1}\right)=\Delta^{-1} A \frac{\partial \psi\left(x_{1}\right)}{\partial x} \geqslant \Delta^{-1} A \frac{\partial \psi\left(x_{2}\right)}{\partial x}=F\left(x_{2}\right)$. Since $F(x)$ is nonnegative $\forall x \in \mathbb{R}_{+}^{n}$ and irreducible $\forall x \in \mathbb{R}_{+}^{n}$, necessarily $F\left(x_{1}\right) \neq F\left(x_{2}\right)$, hence we can apply Theorem 2.14 of [4] from which it follows that $\rho\left(F\left(x_{1}\right)\right)>$ $\rho\left(F\left(x_{2}\right)\right)$.

\section{J. Proof of Proposition 8}

When $\pi=1$ at $x=0$, system (15) represents a consensustype dynamics with Laplacian $L=\Delta-A$. For $L$, the Geršgorin theorem [16] affirms that the eigenvalues of $L$ are located in the union of the $n$ disks

$$
\left\{s \in \mathbb{C} \text { s.t. }\left|s-\delta_{i}\right| \leqslant \sum_{j=1}^{n} a_{i j}\right\} \text {. }
$$


Such disks that are contained in the right half of the complex plane and must all touch the origin. In general, for (15) the inequality (2) holds. When looking at $I-\hat{A}$, then (60) becomes

$$
\left\{s \in \mathbb{C} \text { s.t. }|s-1| \leqslant \sum_{j=1}^{n} \frac{a_{i j}}{\delta_{i}}=1\right\},
$$

i.e., all disks of $I-\hat{A}$ are centered in 1 and have radius 1 , hence in Lemma 1 (1) must hold and $\rho(\hat{A})=1$. If we now consider the case $\pi<1$, then all disks of $I-\pi \hat{A}$ are still centered at 1 but have radius strictly less than 1 , hence, necessarily, $\rho(F(0))=\rho(\pi \hat{A})=\pi \rho(\hat{A})<1$, i.e., condition 1 of Theorem 5 applies, meaning that $x^{*}=0$ is the only asymptotically stable equilibrium. Similarly, when $\pi>1$, all Geršgorin disks of $I-\pi \hat{A}$ are still centered in 1 but have radius strictly larger than 1 . Since by construction (1) of Lemma 1 must hold, it must be $\rho(F(0))=\pi \rho(\hat{A})>1$. Furthermore, Theorem 5 and Proposition 4 hold in this case, and $\rho(F(x)) \rightarrow 0$ for $\|x\| \rightarrow \infty$. Hence condition 2 of Theorem 5 applies, meaning that (15) admits a unique positive fixed point $x^{*} \in \operatorname{int}\left(\mathbb{R}_{+}^{n}\right)$ which is asymptotically stable.

\section{K. Proof of Proposition 9}

By construction, $A$ irreducible implies $\phi(x)$ irreducible everywhere in $\mathbb{R}_{+}^{n}$. Since $p>0$ and $\psi_{i}(0)=0, \phi(0)>0$. From Corollary 3, if $\rho(\Phi(0))<1$, then $\rho(\Phi(x))<1$ everywhere in $\mathbb{R}_{+}^{n}$. Hence Proposition 6 holds, and the claim follows.

\section{Proof of Proposition 10}

System (18) with feedback law (10) can be rewritten as

$$
\dot{x}=K\left(-x+K^{-1} A \psi(x)\right)=K(-x+\hat{A} \psi(x)) .
$$

Condition 1 can then be reformulated as $1>\sum_{j} \frac{a_{i j}}{k_{i}} \forall i=$ $1, \ldots, n$, or, in matrix form

$$
1>\hat{A} \mathbf{1} \text {. }
$$

From Lemma 1, it follows that $1>\rho(\hat{A})$. System (62) is in the form (7) with $\Delta=K$ and $f(x)=\hat{A} \psi(x)$. This implies $F(x)=\hat{A} \frac{\partial \psi(x)}{\partial x}$ and $F(0)=\hat{A}$, from which we have $\rho(F(0))<1$, hence condition 1 of Theorem 5 is applicable and the claim follows. As for condition 2, an analogous argument leads to $\mathbf{1}<\hat{A} \mathbf{1}$, i.e., $\rho(F(0))=\rho(\hat{A})>1$. Combined with Propositions 4 and 7 , this means that the case 2 of Theorem 5 is applicable and leads to existence of $x^{*}>0$ asymptotically stable.

\section{REFERENCES}

[1] Dirk Aeyels and Patrick De Leenheer. Extension of the PerronFrobenius theorem to homogeneous systems. SIAM J. Control Optim., 41(2):563-582, February 2002.

[2] C. Altafini. Dynamics of opinion forming in structurally balanced social networks. PLoS ONE, 7(6):e38135, 2012.

[3] C. Altafini and G. Lini. Predictable dynamics of opinion forming for networks with antagonistic interactions. Automatic Control, IEEE Transactions on, 60(2):342-357, Feb 2015.
[4] A. Berman and R.J. Plemmons. Nonnegative matrices in the mathematical sciences. Classics in applied mathematics. Society for Industrial and Applied Mathematics, 1994.

[5] Vahid S. Bokharaie, Oliver Mason, and Fabian Wirth. Stability and positivity of equilibria for subhomogeneous cooperative systems. Nonlinear Analysis: Theory, Methods \& Applications, 74(17):6416 - 6426, 2011.

[6] P. J. Bushell. Hilbert's metric and positive contraction mappings in a banach space. Archive for Rational Mechanics and Analysis, 52(4):330 338, 1973.

[7] P. De Leenheer and D. Aeyels. Stability properties of equilibria of classes of cooperative systems. Automatic Control, IEEE Transactions on, 46(12):1996-2001, Dec 2001.

[8] L. Farina and S. Rinaldi. Positive Linear Systems: Theory and Applications. A Wiley-Interscience publication. Wiley, 2000.

[9] H. R. Feyzmahdavian, T. Charalambous, and M. Johansson. Subhomogeneous positive monotone systems are insensitive to heterogeneous time-varying delays. In Proc. 21st International Symposium on Mathematical Theory of Networks and Systems (MTNS), 2014.

[10] H.R. Feyzmahdavian, T. Charalambous, and M. Johansson. Stability and performance of continuous-time power control in wireless networks. Automatic Control, IEEE Transactions on, 59(8):2012-2023, Aug 2014.

[11] G.J. Foschini and Z. Miljanic. A simple distributed autonomous power control algorithm and its convergence. Vehicular Technology, IEEE Transactions on, 42(4):641-646, Nov 1993.

[12] A. Franci, V. Srivastava, and N. Ehrich Leonard. A Realization Theory for Bio-inspired Collective Decision-Making. ArXiv e-prints, March 2015.

[13] W.M. Haddad, V.S. Chellaboina, and Q. Hui. Nonnegative and Compartmental Dynamical Systems. Princeton University Press, 2010.

[14] S. Haykin. Neural Networks: A Comprehensive Foundation. International edition. Prentice Hall, 1999.

[15] Josef Hofbauer and Karl Sigmund. Evolutionary games and population dynamics. Cambridge University Press, 1998.

[16] R.A. Horn and C. R. Johnson. Matrix Analysis. Cambdridge University Press, 1985

[17] K.J. Hunt, D. Sbarbaro, R. Zbikowski, and P.J. Gawthrop. Neural networks for control systems - a survey. Automatica, 28(6):1083 1112, 1992.

[18] John A. Jacquez and Carl P. Simon. Qualitative theory of compartmental systems. SIAM Review, 35(1):43-79, 1993.

[19] Eugenius Kaszkurewicz and Amit Bhaya. Matrix diagonal stability in systems and computation. Birkhäuser, Boston, 2000.

[20] John Kennan. Uniqueness of positive fixed points for increasing concave functions on $\mathbb{R}^{n}$ : An elementary result. Review of Economic Dynamics, 4(4):893 - 899, 2001.

[21] A. Khanafer, T. Basar, and B. Gharesifard. Stability properties of infection diffusion dynamics over directed networks. In Decision and Control (CDC), 2014 IEEE 53rd Annual Conference on, pages 62156220, Dec 2014.

[22] Ulrich Krause. Positive nonlinear systems in economics. In Toru Maruyama and Wataru Takahashi, editors, Nonlinear and Convex Analysis in Economic Theory, volume 419 of Lecture Notes in Economics and Mathematical Systems, pages 181-195. Springer Berlin Heidelberg, 1995.

[23] Ulrich Krause and Peter Ranft. A limit set trichotomy for monotone nonlinear dynamical systems. Nonlinear Anal., 19(4):375-392, August 1992.

[24] Chunguang Li, Luonan Chen, and K. Aihara. Stability of genetic networks with SUM regulatory logic: Lur'e system and LMI approach. Circuits and Systems I: Regular Papers, IEEE Transactions on, 53(11):2451-2458, Nov 2006.

[25] W. Lohmiller and J-J. E. Slotine. On contraction analysis for non-linear systems. Automatica, 34(6):683 - 696, 1998.

[26] J. D. Murray. Mathematical biology. Springer-Verlag, Berlin, DEU, 2nd edition, 1993.

[27] Carlo Piccardi and Sergio Rinaldi. Remarks on excitability, stability and sign of equilibria in cooperative systems. Systems \& Control Letters, 46(3): 153 - 163, 2002.

[28] S. U. Pillai, T. Suel, and Seunghun Cha. The perron-frobenius theorem: some of its applications. IEEE Signal Processing Magazine, 22(2):6275, March 2005.

[29] D.D. Siljak. Large-Scale Dynamic Systems: Stability and Structure. North-Holland, 1978.

[30] Hal L. Smith. On the asymptotic behavior of a class of deterministic models of cooperating species. SIAM Journal on Applied Mathematics, 46(3):368-375, 1986 
[31] Hal L. Smith. Monotone Dynamical Systems: An Introduction to the Theory of Competitive and Cooperative Systems, volume 41 of Mathematical Surveys and Monographs. AMS, Providence, RI, 1995.

[32] H.L. Smith. Cooperative systems of differential equations with concave nonlinearities. Nonlinear Analysis: Theory, Methods \& Applications, 10(10): 1037 - 1052, 1986.

[33] E.D. Sontag. Structure and stability of certain chemical networks and applications to the kinetic proofreading model of t-cell receptor signal transduction. Automatic Control, IEEE Transactions on, 46(7):1028 1047, jul 2001.

[34] P Ugo Abara, F. Ticozzi, and C. Altafini. Existence, uniqueness and stability properties of positive equilibria for a class of nonlinear cooperative systems. In 54th IEEE Conference on Decision and Control, Osaka, Japan, 2015

[35] P. Ugo Abara, F. Ticozzi, and C. Altafini. An infinitesimal characterization of nonlinear contracting interference functions. In 55th IEEE Conference on Decision and Control, Las Vegas, NV, 2016.

[36] R.D. Yates. A framework for uplink power control in cellular radio systems. Selected Areas in Communications, IEEE Journal on, 13(7):13411347, Sep 1995.

[37] Huaguang Zhang, Zhanshan Wang, and Derong Liu. A comprehensive review of stability analysis of continuous-time recurrent neural networks. Neural Networks and Learning Systems, IEEE Transactions on, 25(7):1229-1262, July 2014.

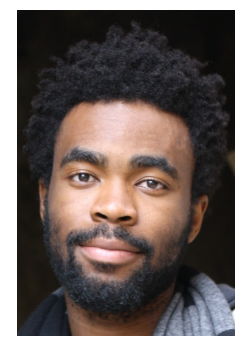

Precious Ugo Abara received the B.S. in Information Engineering and the M.S. in Automation Engineering (summa cum laude) in 2012 and 2014, respectively, from the University of Padova, Italy. He was a visiting researcher at Linkping University from April 2014 to July 2014. He is currently employed as a Marie Curie ESR at the Chair of Information-Oriented Control at the Technical University of Munich, Germany. His research interests include stochastic control, optimization of largescale Cyber-Physical Systems, model predictive control, positive systems, networked controlled systems.

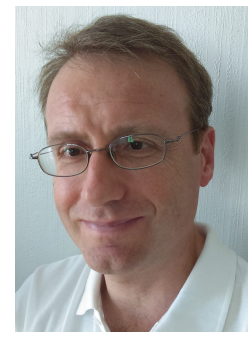

Claudio Altafini received a Master degree ("Laurea") in Electrical Engineering from the University of Padova, Italy, in 1996 and a PhD in Optimization and Systems Theory from the Royal Institute of Technology, Stockholm, Sweden in 2001. From 2001 till 2013 he was with the International School for Advanced Studies (SISSA) in Trieste, Italy. Since 2014 he is a Professor in the Division of Automatic Control, Dept. of Electrical Engineering at Linköping University, Sweden. During 2013-15 he served as an Associate Editor for the IEEE Trans. on Automatic Control. His research interests are in the areas of nonlinear systems analysis and control, with applications to quantum mechanics, systems biology and complex networks.

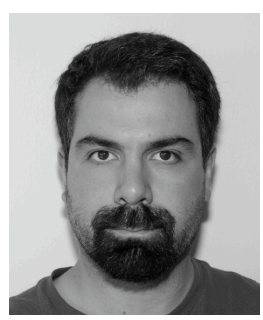

Francesco Ticozzi received the "Laurea" degree in management engineering and a Ph.D. in automatic control and operations research from the University of Padua, Italy, in 2002 and 2007, respectively. Since February 2007, he has been with the Department of Information Engineering at the University of Padova, first as a Research Associate, and then as an Assistant Professor (Ricercatore). During 2005-2010 he held visiting appointments at the Physics and Astronomy Dept. of Dartmouth College, Hanover, New Hampshire, where he is an adjunct assistant professor since July 2011. Dr. Ticozzi's research interests include modeling and control of classical and quantum systems over networks, protection and communication of quantum information, and information-theoretic approaches to control systems. 